\title{
Stereoselective Total Synthesis of (-)-Perrottetinene and Assignment of Its Absolute Configuration
}

Yanling Song, ${ }^{\dagger}{ }^{\star}$ Soonho Hwang, ${ }^{\dagger}$ Ping Gong, ${ }^{\star}$ Deukjoon Kim,${ }^{\dagger}$ and Sanghee Kim*, ${ }^{\dagger}$

${ }^{\dagger}$ College of Pharmacy, Seoul National University, San 56-1, Shilim, Kwanak, Seoul 151-742, Korea, and ${ }^{\ddagger}$ School of Pharmaceutical Engineering, Shenyang Pharmaceutical University, Liaoning 110016, P. R. China

pennkim@snu.ac.kr

\section{Contents}

General Experimental Method

Full experimental procedures and analytical data of compounds

${ }^{1} \mathrm{H}$ NMR and ${ }^{13} \mathrm{C}$ NMR spectra of compound 9

S11-S12

${ }^{1} \mathrm{H}$ NMR and ${ }^{13} \mathrm{C}$ NMR spectra of compound $\mathbf{1 0}$

S13-S14

${ }^{1} \mathrm{H}$ NMR and ${ }^{13} \mathrm{C}$ NMR spectra of compound $\mathbf{1 1}$

S15-S16

${ }^{1} \mathrm{H}$ NMR and ${ }^{13} \mathrm{C}$ NMR spectra of compound $\mathbf{1 2}$

S17-S18

${ }^{1} \mathrm{H}$ NMR and ${ }^{13} \mathrm{C}$ NMR spectra of compound $\mathbf{1 3}$

S19-S20

${ }^{1} \mathrm{H}$ NMR and ${ }^{13} \mathrm{C}$ NMR spectra of compound $\mathbf{1 4}$

$\mathrm{S} 21-\mathrm{S} 22$

${ }^{1} \mathrm{H}$ NMR and ${ }^{13} \mathrm{C}$ NMR spectra of compound $\mathbf{1 5}$

S23-S24

${ }^{1} \mathrm{H}$ NMR and ${ }^{13} \mathrm{C}$ NMR spectra of compound $\mathbf{1 7}$

S25-S26

${ }^{1} \mathrm{H}$ NMR and ${ }^{13} \mathrm{C}$ NMR spectra of compound 3

S27-S28 
General. All chemicals were reagent grade and used as purchased. All reactions were performed under an inert atmosphere of dry argon or nitrogen using distilled dry solvents. Reactions were monitored by TLC analysis using silica gel 60 F-254 thin layer plates. Melting points are uncorrected. Flash column chromatography was carried out on silica gel (230-400 mesh). Optical rotations were measured using sodium light (D line $589.3 \mathrm{~nm}$ ). ${ }^{1} \mathrm{H}$ NMR and ${ }^{13} \mathrm{C}$ NMR spectra were recorded in $\delta$ units relative to deuterated solvent as internal reference by $300 \mathrm{MHz}$ or $400 \mathrm{MHz}$ NMR instrument. IR spectra were measured on Fourier Transform Infrared spectrometer. Mass spectra (MS) were recorded using fast atom bombardment (FAB). High resolution mass spectra (HRMS) were recorded using FAB. Compound $\mathbf{6},{ }^{1} \mathbf{7},{ }^{2}$ and $\mathbf{8}^{3}$ were prepared as previously described.

(1) Ali, M. A.; Kondo, K.; Tsuda, Y. Chem. Pharm. Bull. 1992, 40, 1130-1136.

(2) Lee, T.; Kim, S. Tetrahedron: Asymmetry 2003, 14, 1951-1954.

(3) Smith, A. B.; Toder, B. H.; Branca, S. J.; Dieter, R. K. J. Am. Chem. Soc. 1981, 103, 1996-2008. 
2-Iodo-5-phenethylbenzene-1,3-diol (9)<smiles>Oc1cc(O)cc(CCc2ccccc2)c1</smiles>

6

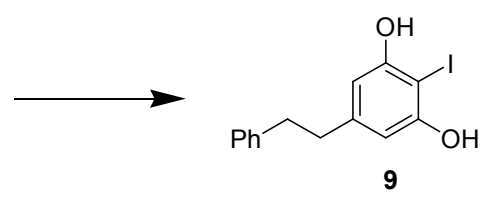

To a mixture of $6(1.78 \mathrm{~g}, 8.30 \mathrm{mmol})$ and $\mathrm{I}_{2}(2.32 \mathrm{~g}, 9.12 \mathrm{mmol})$ in THF $(100 \mathrm{~mL})$ and $\mathrm{H}_{2} \mathrm{O}(100 \mathrm{~mL})$ was slowly added $\mathrm{NaHCO}_{3}(0.77 \mathrm{~g}, 9.12 \mathrm{mmol})$ in portions at $0{ }^{\circ} \mathrm{C}$ with vigorous stirring. The reaction mixture was warmed to room temperature over 10 min and continued to stir for additional $10 \mathrm{~min}$. The mixture was extracted with EtOAc three times. The combined organic layers were washed with brine, dried over $\mathrm{MgSO}_{4}$ and concentrated. This residue was purified by column chromatography on silica gel (Hexane/EtOAc, 5:1) to give $9(2.40 \mathrm{~g}, 85 \%)$ as a white solid: $\mathrm{mp} 110.6-111.3{ }^{\circ} \mathrm{C} ;{ }^{1} \mathrm{H}$ NMR $\left(\mathrm{CDCl}_{3}, 300 \mathrm{MHz}\right) \delta 2.77(\mathrm{~m}, 4 \mathrm{H}), 5.20$ (br s, 2H), $6.33(\mathrm{~s}, 2 \mathrm{H}), 7.11(\mathrm{~m}, 3 \mathrm{H})$, $7.20(\mathrm{~m}, 2 \mathrm{H}) ;{ }^{13} \mathrm{C} \mathrm{NMR}\left(\mathrm{CDCl}_{3}, 100 \mathrm{MHz}\right) \delta 37.2,37.3,74.2,107.6,126.0,128.4$, 141.2, 145.0, 155.3; ${ }^{13} \mathrm{C} \mathrm{NMR}\left(\mathrm{CD}_{3} \mathrm{OD}, 125 \mathrm{MHz}\right) \delta 39.4,39.5,72.9,108.2,127.6$, 130.0, 130.2, 143.6, 145.8, 159.7; IR $\left(\mathrm{CHCl}_{3}\right) \cup_{\max } 3418,3229,3024,2926,2858,1579$ $\left(\mathrm{cm}^{-1}\right)$; MS (FAB) $(\mathrm{m} / \mathrm{z}) 340\left(\mathrm{M}^{+}, 30\right), 154$ (100), 136 (65), 307 (32); HRMS (FAB) calcd for $\mathrm{C}_{14} \mathrm{H}_{14} \mathrm{IO}_{2} 341.0039\left([\mathrm{M}+\mathrm{H}]^{+}\right)$, found 341.0041 .

\section{2-Iodo-5-phenethyl-1,3-phenylene dibenzoate (10)}

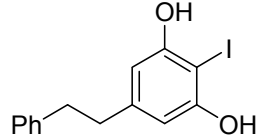

9

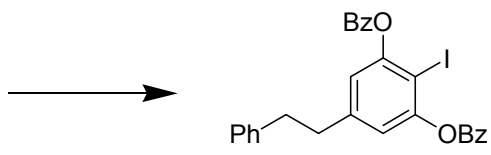

10

To a solution of $9(2.40 \mathrm{~g}, 7.06 \mathrm{mmol})$ in THF $(70 \mathrm{~mL})$ was added $\mathrm{NaH}(622 \mathrm{mg}, 15.5$ mmol, $60 \%$ dispersion in mineral oil) in portions at $0{ }^{\circ} \mathrm{C}$. After the mixture was stirred for $10 \mathrm{~min}$, benzoyl chloride $(1.8 \mathrm{~mL}, 15.5 \mathrm{mmol})$ was added. This reaction mixture was 
allowed to warm to room temperature and continued to stir for $3 \mathrm{~h}$. The reaction was quenched with saturated aqueous $\mathrm{NH}_{4} \mathrm{Cl}$ at $0{ }^{\circ} \mathrm{C}$. The mixture was extracted with EtOAc three times. The combined organic layers were washed with brine, dried over $\mathrm{MgSO}_{4}$ and concentrated. This residue was purified by column chromatography on silica gel (hexane/EtOAc, 10:1) to give $\mathbf{1 0}(3.80 \mathrm{~g}, 98 \%)$ as a white solid: mp $119.7-121.3{ }^{\circ} \mathrm{C} ;{ }^{1} \mathrm{H}$ NMR $\left(\mathrm{CDCl}_{3}, 300 \mathrm{MHz}\right) \delta 2.97(\mathrm{~s}, 4 \mathrm{H}), 7.05(\mathrm{~s}, 2 \mathrm{H}), 7.20(\mathrm{~m}, 3 \mathrm{H}), 7.27(\mathrm{~m}, 2 \mathrm{H}), 7.53$ (m, 4H), $7.66(\mathrm{~m}, 2 \mathrm{H}), 8.27(\mathrm{~d}, J=8.3 \mathrm{~Hz}, 4 \mathrm{H}) ;{ }^{13} \mathrm{C} \mathrm{NMR}\left(\mathrm{CDCl}_{3}, 100 \mathrm{MHz}\right) \delta 37.1$, $37.4,84.7,120.7,126.2,128.4,128.5,128.7,129.0,130.5,133.9,140.9,144.3,152.4$, 164.1; IR $\left(\mathrm{CHCl}_{3}\right) \cup_{\max } 3063,2926,1743,1246,1084,1064\left(\mathrm{~cm}^{-1}\right) ;$ MS (FAB) $(\mathrm{m} / \mathrm{z})$ $549\left([\mathrm{M}+1]^{+}, 25\right), 105$ (100), 77 (18); HRMS (FAB) calcd $\mathrm{C}_{28} \mathrm{H}_{22} \mathrm{IO}_{4} 549.0563$ $\left([\mathrm{M}+\mathrm{H}]^{+}\right)$, found 549.0577 .

(S)-(E)-2-(3-Hydroxybut-1-enyl)-5-phenethyl-1,3-phenylene dibenzoate (11)

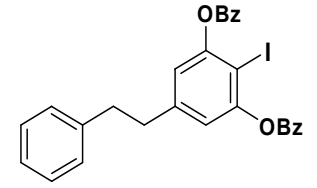

10

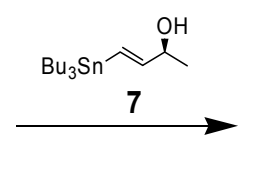

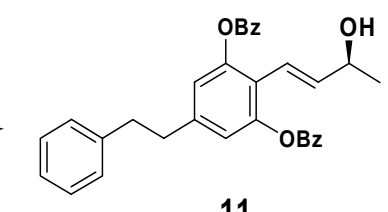

11

To a solution of $10(3.16 \mathrm{~g}, 5.76 \mathrm{mmol})$ and $(S)-(E)$-4-(tributylstannyl)but-3-en-2-ol (7) $(2.50 \mathrm{~g}, 6.92 \mathrm{mmol})$ in toluene $(60 \mathrm{~mL})$ were added $\mathrm{Pd}_{2}(\mathrm{dba})_{3}(300 \mathrm{mg}, 0.288$ mmol), $\mathrm{P}(t-\mathrm{Bu})_{3}(117 \mathrm{mg}, 0.576 \mathrm{mmol}), \mathrm{CuI}(110 \mathrm{mg}, 0.576 \mathrm{mmol})$, and $\mathrm{CsF}(1.92 \mathrm{~g}$, $12.7 \mathrm{mmol}$ ) under $\mathrm{N}_{2}$ atmosphere. The mixture was heated to $80{ }^{\circ} \mathrm{C}$ for $5 \mathrm{~h}$. After the mixture was cooled to room temperature, it was diluted with EtOAc and then filtrated through Celite and concentrated in vacuo. The residue was purified by column chromatography on silica gel (hexane/EtOAc, 5:1 to 2:1) to give the desired compound $(2.21 \mathrm{~g}, 78 \%)$ as an oil: $[\alpha]^{25} \mathrm{D}+2.7\left(c 0.55, \mathrm{CHCl}_{3}\right) ;{ }^{1} \mathrm{H} \mathrm{NMR}\left(\mathrm{CDCl}_{3}, 300 \mathrm{MHz}\right) \delta 0.95$ 
$(\mathrm{d}, J=6.4 \mathrm{~Hz}, 3 \mathrm{H}), 1.54$ (br s, 1H), $2.98(\mathrm{~s}, 4 \mathrm{H}), 4.17$ (dt, $J=6.2,6.4 \mathrm{~Hz}, 1 \mathrm{H}), 6.24$ (dd, $J=6.2,16.2 \mathrm{~Hz}, 1 \mathrm{H}), 6.43(\mathrm{~d}, J=16.2 \mathrm{~Hz}, 1 \mathrm{H}), 7.02(\mathrm{~s}, 2 \mathrm{H}), 7.24(\mathrm{~m}, 3 \mathrm{H}), 7.32(\mathrm{~m}$, 2H), $7.54(\mathrm{~m}, 4 \mathrm{H}), 7.67(\mathrm{~m}, 2 \mathrm{H}), 8.22(\mathrm{~d}, J=8.0 \mathrm{~Hz}, 4 \mathrm{H}) ;{ }^{13} \mathrm{C} \mathrm{NMR}\left(\mathrm{CDCl}_{3}, 100 \mathrm{MHz}\right)$ $\delta 22.8,37.1,37.3,68.9,118.2,120.5,121.7,126.0,128.3,128.4,128.6,129.1,130.1$, 133.7, 140.2, 141.1, 142.4, 149.2, 164.6; IR $\left(\mathrm{CHCl}_{3}\right) \cup_{\max } 3447,3028,2970,1738$, 1248, $1086\left(\mathrm{~cm}^{-1}\right)$; MS (FAB) $(\mathrm{m} / \mathrm{z}) 515\left([\mathrm{M}+23]^{+}, 10\right), 154(100), 136(68), 105(60)$, 307 (30); HRMS (FAB) calcd $\mathrm{C}_{32} \mathrm{H}_{28} \mathrm{O}_{5} \mathrm{Na} 515.1834\left([\mathrm{M}+\mathrm{Na}]^{+}\right)$, found 515.1829.

\section{(S)-(E)-2-(3-(5-Methylhex-5-enoyloxy)but-1-enyl)-5-phenethyl-1,3-phenylene}

\section{dibenzoate (12)}

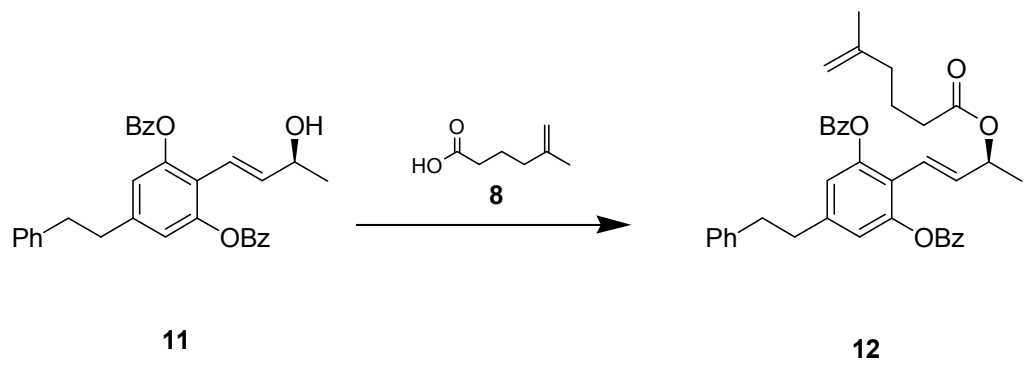

To a solution of alcohol $11(900 \mathrm{mg}, 1.82 \mathrm{mmol})$ in $\mathrm{CH}_{2} \mathrm{Cl}_{2}(40 \mathrm{~mL})$ were added 5methyl-hex-5-enoic acid (8) (350 mg, $2.74 \mathrm{mmol})$, DMAP (66.3 mg, $0.546 \mathrm{mmol}$ ), and DCC (566 mg, $2.74 \mathrm{mmol})$ at $0{ }^{\circ} \mathrm{C}$. The reaction mixture was stirred for $16 \mathrm{~h}$ at room temperature followed by dilution with hexane. The generated white precipitate was removed by filtration through Celite. The filtrate was concentrated and the residue was purified by column chromatography on silica gel (hexane/EtOAc, 15:1) to give 12 (1.07 g, 98\%) as an oil: $[\alpha]^{25}-35.9\left(c 1.89, \mathrm{CHCl}_{3}\right) ;{ }^{1} \mathrm{H} \mathrm{NMR}\left(\mathrm{CDCl}_{3}, 400 \mathrm{MHz}\right) \delta 0.98(\mathrm{~d}, J$ $=6.5 \mathrm{~Hz}, 3 \mathrm{H}), 1.50(\mathrm{~m}, 2 \mathrm{H}), 1.64(\mathrm{~s}, 3 \mathrm{H}), 1.84(\mathrm{~m}, 3 \mathrm{H}), 1.95(\mathrm{~m}, 1 \mathrm{H}), 2.95(\mathrm{~s}, 4 \mathrm{H}), 4.59$ (s, 1H), $4.68(\mathrm{~s}, 1 \mathrm{H}), 5.23(\mathrm{dt}, J=6.2,6.5 \mathrm{~Hz}, 1 \mathrm{H}), 6.14(\mathrm{dd}, J=6.3,16.3 \mathrm{~Hz}, 1 \mathrm{H}), 6.42$ $(\mathrm{d}, J=16.3 \mathrm{~Hz}, 1 \mathrm{H}), 6.99(\mathrm{~s}, 2 \mathrm{H}), 7.18(\mathrm{~m}, 3 \mathrm{H}), 7.28(\mathrm{~m}, 2 \mathrm{H}), 7.51(\mathrm{~m}, 4 \mathrm{H}), 7.63(\mathrm{~m}$, 
2H), $8.18(\mathrm{~d}, J=7.3 \mathrm{~Hz}, 4 \mathrm{H}) ;{ }^{13} \mathrm{C} \mathrm{NMR}\left(\mathrm{CDCl}_{3}, 100 \mathrm{MHz}\right) \delta 19.9,22.0,22.4,33.4$, $36.8,37.1,37.3,70.2,110.5,120.2,120.5,121.3,126.0,128.3,128.3,128.6,129.1$, 130.0, 133.6, 135.3, 141.0, 142.6, 144.5, 149.1, 164.4, 172.2; IR $\left(\mathrm{CHCl}_{3}\right) \mathrm{U}_{\max } 3065$, 2932, 1739, 1246, $1086\left(\mathrm{~cm}^{-1}\right)$; MS (FAB) $(\mathrm{m} / \mathrm{z}) 625\left([\mathrm{M}+23]^{+}, 8\right), 105(100), 154(45)$, 475 (30); HRMS (FAB) calcd $\mathrm{C}_{39} \mathrm{H}_{38} \mathrm{O}_{6} \mathrm{Na} 625.2566\left([\mathrm{M}+\mathrm{Na}]^{+}\right)$, found 625.2567.

(2S,3R)-(E)-3-(2,6-Bis(benzoyloxy)-4-phenethylphenyl)-2-(3-methylbut-3enyl)hex-4-enoic acid (13)

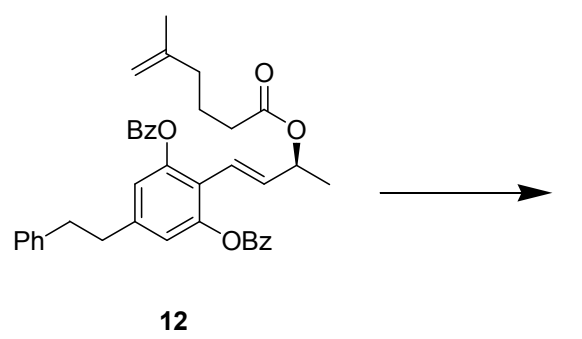

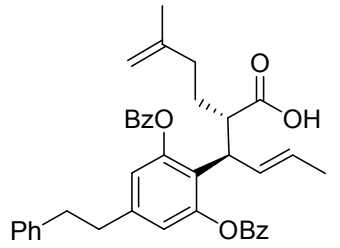

13

To a stirred solution of 12 (600 mg, $1.00 \mathrm{mmol})$ in THF (40 mL) and HMPA (10 mL) was added TBSCl (302 mg, $2.00 \mathrm{mmol})$ in THF (5 mL). After the mixture was cooled to $-78{ }^{\circ} \mathrm{C}$, LDA (4.2 mL, $0.475 \mathrm{M}$ in THF) was slowly added. The reaction mixture was slowly warmed to room temperature and stirred for $15 \mathrm{~h}$. It was acidified to $\mathrm{pH} 2-3$ with $1 \mathrm{~N} \mathrm{HCl}$ aqueous solution and extracted with EtOAc twice. The combined organic layers were washed with brine, dried over $\mathrm{Na}_{2} \mathrm{SO}_{4}$, and concentrated. The residue was purified by column chromatography on silica gel (hexane/EtOAc, 3:1) to give 13 (360 mg, 60\%) as an oil: $[\alpha]^{25}-3.3\left(c 1.07, \mathrm{CHCl}_{3}\right) ;{ }^{1} \mathrm{H} \mathrm{NMR}\left(\mathrm{CDCl}_{3}, 300 \mathrm{MHz}\right) \delta 1.36(\mathrm{~d}$, $J=6.2 \mathrm{~Hz}, 3 \mathrm{H}), 1.50(\mathrm{~m}, 2 \mathrm{H}), 1.56(\mathrm{~s}, 3 \mathrm{H}), 1.90(\mathrm{~m}, 2 \mathrm{H}), 2.93(\mathrm{~s}, 4 \mathrm{H}), 3.05(\mathrm{dt}, J=3.7$, 10.6 Hz, 1H), 3.92 (dd, $J=7.2,11.6 \mathrm{~Hz}, 1 \mathrm{H}), 4.60$ (s, 1H), 4.67 (s, 1H), $5.34(\mathrm{~m}, 1 \mathrm{H})$, $5.52(\mathrm{dd}, J=7.3,15.4 \mathrm{~Hz}, 1 \mathrm{H}), 6.97(\mathrm{~s}, 2 \mathrm{H}), 7.17(\mathrm{~m}, 3 \mathrm{H}), 7.27(\mathrm{~m}, 2 \mathrm{H}), 7.51(\mathrm{~m}, 4 \mathrm{H})$, $7.64(\mathrm{~m}, 2 \mathrm{H}), 8.19(\mathrm{~d}, J=8.3 \mathrm{~Hz}, 4 \mathrm{H}) ;{ }^{13} \mathrm{C} \mathrm{NMR}\left(\mathrm{CDCl}_{3}, 100 \mathrm{MHz}\right) \delta 17.8,22.2,28.7$, 
$35.2,37.1,37.4,42.0,48.2,110.8,121.1,124.1,126.0,128.0,128.4,128.7,128.8$, 129.2, 130.2, 133.8, 141.2, 141.8, 144.5, 149.7, 164.7; IR $\left(\mathrm{CHCl}_{3}\right) \cup_{\max } 2926,1741$, 1705, $1244\left(\mathrm{~cm}^{-1}\right)$; MS (FAB) $(\mathrm{m} / \mathrm{z}) 625\left([\mathrm{M}+23]^{+}, 5\right), 154(100), 136(65), 307(30)$, 105 (24); HRMS (FAB) calcd $\mathrm{C}_{39} \mathrm{H}_{38} \mathrm{O}_{6} \mathrm{Na} 625.2566\left([\mathrm{M}+\mathrm{Na}]^{+}\right)$, found 625.2582.

\section{2-((4R,5S)-(E)-5-(Methoxycarbonyl)-8-methylnona-2,8-dien-4-yl)-5-phenethyl-}

\section{1,3-phenylene dibenzoate (14)}

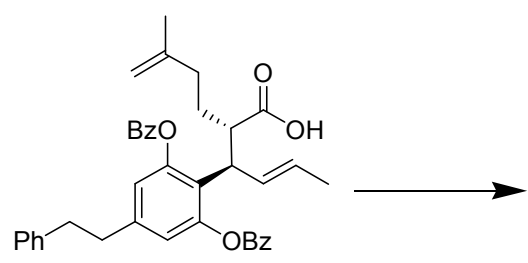

13

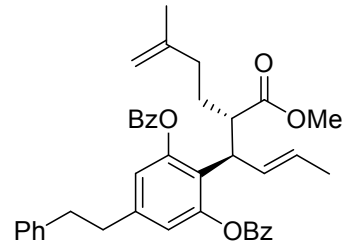

14

To a solution of $\mathbf{1 3}(160 \mathrm{mg}, 0.260 \mathrm{mmol})$ in $\mathrm{CH}_{2} \mathrm{Cl}_{2}(4.0 \mathrm{~mL})$ and $\mathrm{MeOH}(1.2 \mathrm{~mL})$ were added DMAP $(9.7 \mathrm{mg}, 0.080 \mathrm{mmol})$ and DCC $(86 \mathrm{mg}, 0.40 \mathrm{mmol})$. The reaction mixture was stirred for $16 \mathrm{~h}$ at room temperature followed by dilution with hexane. The generated white precipitate was removed by filtration through Celite. The filtrate was concentrated and the residue was purified by column chromatography on silica gel (hexane/EtOAc, 15:1) to give $\mathbf{1 4}(148 \mathrm{mg}, 91 \%)$ as an oil: $[\alpha]^{25}+17.1\left(c 0.95, \mathrm{CHCl}_{3}\right)$; ${ }^{1} \mathrm{H} \mathrm{NMR}\left(\mathrm{CDCl}_{3}, 400 \mathrm{MHz}\right) \delta 1.37(\mathrm{~d}, J=6.2 \mathrm{~Hz}, 3 \mathrm{H}), 1.43(\mathrm{~m}, 1 \mathrm{H}), 1.54(\mathrm{~s}, 3 \mathrm{H}), 1.59$ (m, 1H), $1.80(\mathrm{~m}, 2 \mathrm{H}), 2.90(\mathrm{~s}, 4 \mathrm{H}), 3.03(\mathrm{dt}, J=3.3,11.0 \mathrm{~Hz}, 1 \mathrm{H}), 3.49$ (s, 3H), 3.90 (dd, $J=7.8,10.6 \mathrm{~Hz}, 1 \mathrm{H}), 4.55(\mathrm{~s}, 1 \mathrm{H}), 4.63(\mathrm{~s}, 1 \mathrm{H}), 5.29(\mathrm{~m}, 1 \mathrm{H}), 5.48(\mathrm{dd}, J=7.4$, 15.2 Hz, 1H), $7.94(\mathrm{~s}, 2 \mathrm{H}), 7.13(\mathrm{~m}, 3 \mathrm{H}), 7.22(\mathrm{~m}, 2 \mathrm{H}), 7.50(\mathrm{~m}, 4 \mathrm{H}), 7.62(\mathrm{~m}, 2 \mathrm{H}), 8.18$ $(\mathrm{d}, J=7.7 \mathrm{~Hz}, 4 \mathrm{H}) ;{ }^{13} \mathrm{C} \mathrm{NMR}\left(\mathrm{CDCl}_{3}, 100 \mathrm{MHz}\right) \delta 17.8,22.2,28.8,35.4,37.1,37.4$, $42.5,48.5,51.2,110.5,121.0,124.4,126.0,127.7,128.4,128.7,129.1,129.3,130.2$ 133.8, 141.3, 141.7, 144.7, 149.7, 164.8, 175.6; IR $\left(\mathrm{CHCl}_{3}\right) \cup_{\max } 2932,1739,1244$, 
1086, $1066\left(\mathrm{~cm}^{-1}\right)$; MS (FAB) $(\mathrm{m} / \mathrm{z}) 617\left([\mathrm{M}+1]^{+}, 10\right), 105$ (100), 154 (45); HRMS (FAB) calcd $\mathrm{C}_{40} \mathrm{H}_{41} \mathrm{O}_{6} 617.2903\left([\mathrm{M}+\mathrm{H}]^{+}\right)$, found 617.2892.

\section{2-((4R,5S)-(E)-5-(2-Hydroxypropan-2-yl)-8-methylnona-2,8-dien-4-yl)-5-}

\section{phenethylbenzene-1,3-diol (15)}

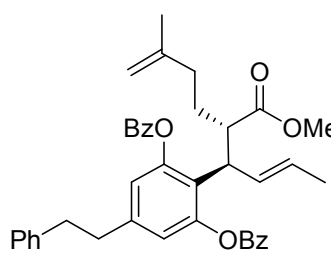

14

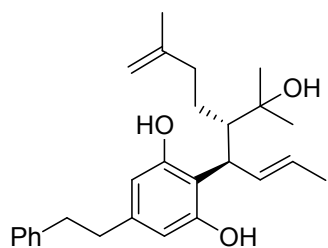

15

To a solution of $14(120 \mathrm{mg}, 0.200 \mathrm{mmol})$ in THF $(6.0 \mathrm{~mL})$ was slowly added $\mathrm{CH}_{3} \mathrm{MgBr}(1.96 \mathrm{~mL}, 3.0 \mathrm{M}$ in ether, $5.84 \mathrm{mmol})$ at room temperature. The mixture was heated to reflux for $2 \mathrm{~h}$ and then cooled to room temperature. The reaction was quenched with saturated $\mathrm{NH}_{4} \mathrm{Cl}$ solution, poured into brine, and extracted with ether twice. The combined organic layers were dried over $\mathrm{MgSO}_{4}$ and concentrated. The residue was purified by column chromatography on silica gel (hexane/EtOAc, 5:1) to give $15(72 \mathrm{mg}, 90 \%)$ as an oil: $[\alpha]^{25}{ }_{\mathrm{D}}+50.8\left(\mathrm{c} 1.76, \mathrm{CHCl}_{3}\right) ;{ }^{1} \mathrm{H} \mathrm{NMR}\left(\mathrm{CDCl}_{3}, 400\right.$ MHz) $\delta 1.27(\mathrm{~s}, 3 \mathrm{H}), 1.29(\mathrm{~s}, 3 \mathrm{H}), 1.36(\mathrm{~m}, 2 \mathrm{H}), 1.47(\mathrm{~s}, 3 \mathrm{H}), 1.68(\mathrm{~d}, J=6.3 \mathrm{~Hz}, 3 \mathrm{H})$, $1.86(\mathrm{~m}, 2 \mathrm{H}), 2.38(\mathrm{~m}, 1 \mathrm{H}), 2.78(\mathrm{~m}, 4 \mathrm{H}), 4.06(\mathrm{t}, J=8.2 \mathrm{~Hz}, 1 \mathrm{H}), 4.44(\mathrm{~s}, 1 \mathrm{H}), 4.54(\mathrm{~s}$, 1H), $5.66(\mathrm{~m}, 1 \mathrm{H}), 6.21(\mathrm{~s}, 2 \mathrm{H}), 6.31(\mathrm{dd}, J=1.4,8.4 \mathrm{~Hz}, 1 \mathrm{H}), 7.16(\mathrm{~m}, 3 \mathrm{H}), 7.26(\mathrm{~m}$, $2 \mathrm{H}) ;{ }^{13} \mathrm{C} \mathrm{NMR}\left(\mathrm{CDCl}_{3}, 100 \mathrm{MHz}\right) \delta 18.1,22.0,25.0,29.8,29.9,37.3,37.5,38.7,41.8$, $50.0,108.6,109.5,113.9,125.9,126.8,128.3,128.4,134.6,141.7,141.8,146.5,155.4$; IR $\left(\mathrm{CHCl}_{3}\right) \cup_{\max } 3497,3263,3028,2972,2928,1620,1433\left(\mathrm{~cm}^{-1}\right)$; MS (FAB) $(\mathrm{m} / \mathrm{z}) 408$ $\left(\mathrm{M}^{+}, 8\right), 267$ (100), 154 (30), 136 (20); HRMS (FAB) calcd $\mathrm{C}_{27} \mathrm{H}_{36} \mathrm{O}_{3} 408.2664\left(\mathrm{M}^{+}\right)$, found 408.2677 . 


\section{2-((1R,6S)-6-(2-Hydroxypropan-2-yl)-3-methylcyclohex-2-enyl)-5-}

\section{phenethylbenzene-1,3-diol (17)}

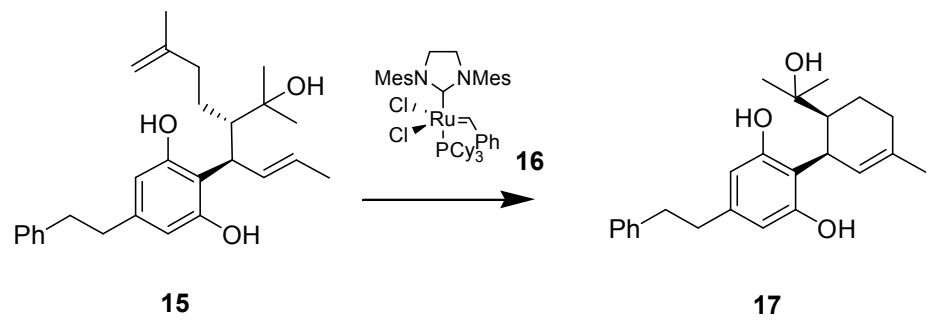

To a solution of the $15(60 \mathrm{mg}, 0.15 \mathrm{mmol})$ in $\mathrm{CH}_{2} \mathrm{Cl}_{2}(10 \mathrm{~mL})$ was added secondgeneration Grubbs' catalyst $(6.0 \mathrm{mg}, 0.0076 \mathrm{mmol})$. The mixture was heated to reflux for $16 \mathrm{~h}$ and then cooled to room temperature. The volatiles were removed, and the residue was purified by flash silica gel column chromatography (hexane/EtOAc, 5:1) to give 17 (49 mg, 90\%) as an oil: $[\alpha]^{25}{ }_{\mathrm{D}}-150.9\left(c\right.$ 1.00, $\left.\mathrm{CHCl}_{3}\right) ;{ }^{1} \mathrm{H}$ NMR $\left(\mathrm{CDCl}_{3}, 400\right.$ MHz) $\delta 0.62(\mathrm{~s}, 3 \mathrm{H}), 1.22(\mathrm{~s}, 3 \mathrm{H}), 1.49(\mathrm{~m}, 1 \mathrm{H}), 1.69(\mathrm{~s}, 3 \mathrm{H}), 1.74(\mathrm{~m}, 1 \mathrm{H}), 1.89(\mathrm{~m}$, $1 \mathrm{H}), 2.15(\mathrm{~d}, J=4.9 \mathrm{~Hz}, 2 \mathrm{H}), 2.65(\mathrm{~m}, 2 \mathrm{H}), 2.73(\mathrm{~m}, 2 \mathrm{H}), 3.87(\mathrm{~s}, 1 \mathrm{H}), 5.63(\mathrm{~d}, J=4.4$ $\mathrm{Hz}, 1 \mathrm{H}), 6.18(\mathrm{~s}, 1 \mathrm{H}), 6.26(\mathrm{~s}, 1 \mathrm{H}), 6.69(\mathrm{br} \mathrm{s}, 1 \mathrm{H}), 7.08(\mathrm{~m}, 3 \mathrm{H}), 7.14(\mathrm{~m}, 2 \mathrm{H}) ;{ }^{13} \mathrm{C}$ $\operatorname{NMR}\left(\mathrm{CDCl}_{3}, 100 \mathrm{MHz}\right) \delta 22.5,23.0,23.3,31.9,32.0,33.1,37.3,37.4,50.5,75.0$, $110.7,110.8,112.3,125.5,125.7,125.8,128.2,128.4,139.1,141.8,143.0,154.9$, 157.3; IR $\left(\mathrm{CHCl}_{3}\right) \cup_{\max } 3410,3028,2943,1630,1448\left(\mathrm{~cm}^{-1}\right)$; MS (FAB) $(\mathrm{m} / \mathrm{z}) 366\left(\mathrm{M}^{+}\right.$, 70), 349 (100), 348 (75), 265 (50); HRMS (FAB) calcd $\mathrm{C}_{24} \mathrm{H}_{30} \mathrm{O}_{3} 366.2195\left(\mathrm{M}^{+}\right)$, found 366.2182 . 


\section{(-)-Perrottetinene (3)}

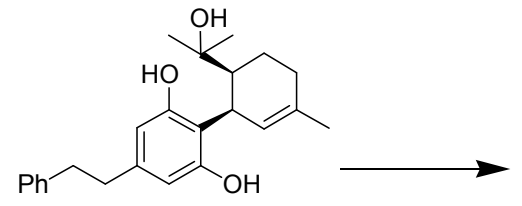

17

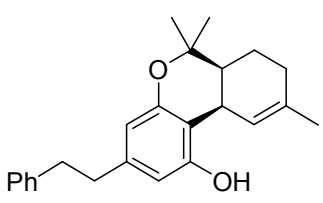

3

To a solution of the $17(24 \mathrm{mg}, 0.065 \mathrm{mmol})$ in benzene $(6.5 \mathrm{~mL})$ was added $\mathrm{TsOH}$ $(0.50 \mathrm{mg}, 0.0015 \mathrm{mmol})$. The mixture was heated to reflux for $0.5 \mathrm{~h}$ and then cooled to room temperature. It was poured into saturated $\mathrm{NaHCO}_{3}$ solution and extracted with EtOAc twice. The combined organic layers were washed with brine, dried over $\mathrm{Na}_{2} \mathrm{SO}_{4}$, and concentrated. The residue was purified by column chromatography on silica gel (hexane/EtOAc, 20:1) to give (-)-perrottetinene (3) $(13 \mathrm{mg}, 55 \%)$ as an oil: $[\alpha]^{25}-$ $118.2\left(c 0.30, \mathrm{CHCl}_{3}\right)\left\{\right.$ lit. $\left.^{4}[\alpha]^{22}{ }_{\mathrm{D}}-121.3\left(c 0.4, \mathrm{CHCl}_{3}\right)\right\} ;{ }^{1} \mathrm{H} \mathrm{NMR}\left(\mathrm{CDCl}_{3}, 400 \mathrm{MHz}\right)$ $\delta 1.26(\mathrm{~s}, 3 \mathrm{H}), 1.38(\mathrm{~s}, 3 \mathrm{H}), 1.46(\mathrm{~m}, 1 \mathrm{H}), 1.69(\mathrm{~s}, 3 \mathrm{H}), 1.73(\mathrm{~m}, 1 \mathrm{H}), 1.91(\mathrm{~m}, 1 \mathrm{H}), 1.96$ (m, 2H), $2.74(\mathrm{~m}, 2 \mathrm{H}), 2.84(\mathrm{~m}, 2 \mathrm{H}), 3.55$ (br s, 1H), 6.14 (d, J=1.3 Hz, 1H), 6.19 (br, 1H), $6.29(\mathrm{~s}, 1 \mathrm{H}), 7.16(\mathrm{~m}, 3 \mathrm{H}), 7.25(\mathrm{~m}, 2 \mathrm{H}) ;{ }^{13} \mathrm{C} \mathrm{NMR}\left(\mathrm{CDCl}_{3}, 100 \mathrm{MHz}\right) \delta 21.1$, $24.1,25.7,26.3,30.2,31.9,37.8,37.8,40.4,76.7,108.3,110.3,110.4,122.2,126.3$, 128.9, 135.6, 141.8, 142.4, 154.4, 155.3; IR $\left(\mathrm{CHCl}_{3}\right) \cup_{\max } 3377,2926,1624,1577,1498$, 1427, 1365, 1157, $1055\left(\mathrm{~cm}^{-1}\right)$; MS (FAB) $(\mathrm{m} / z) 348\left(\mathrm{M}^{+}, 5\right), 154(100), 134(65), 307$ (30); HRMS (FAB) calcd $\mathrm{C}_{24} \mathrm{H}_{28} \mathrm{O}_{2} 348.2089\left(\mathrm{M}^{+}\right)$, found 348.2081.

(4) Toyota, M.; Kinugawa, T.; Asakawa, Y. Phytochemistry 1994, 37, 859-862. 


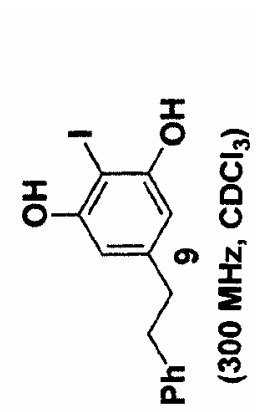

$680 L$ '?

ट9LL

60EL $L$

ESG $L$ '

828L

996L'

$6208^{\circ} \mathrm{C}$

ट $\angle 00^{\circ}$

SLIB.

$1928 \cdot 2$

E66 ' 9

$0 Z^{\prime} 8$

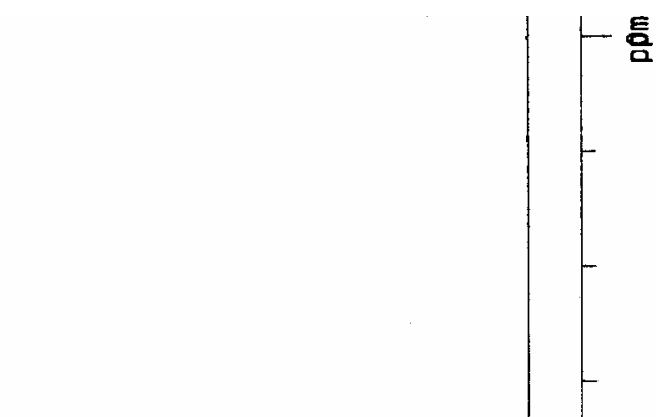

S92E 9

$0960^{\circ} \mathrm{L}$

$600 \mathrm{I}^{\circ} \mathrm{L}$

C9I : L

GDET L

9G9I $L$

$\angle I \angle V^{\circ} L$

$96 \angle T^{\circ} L$

$970 \mathcal{Z}^{\circ} L$

टLCट' $L$

GIE ' $L$
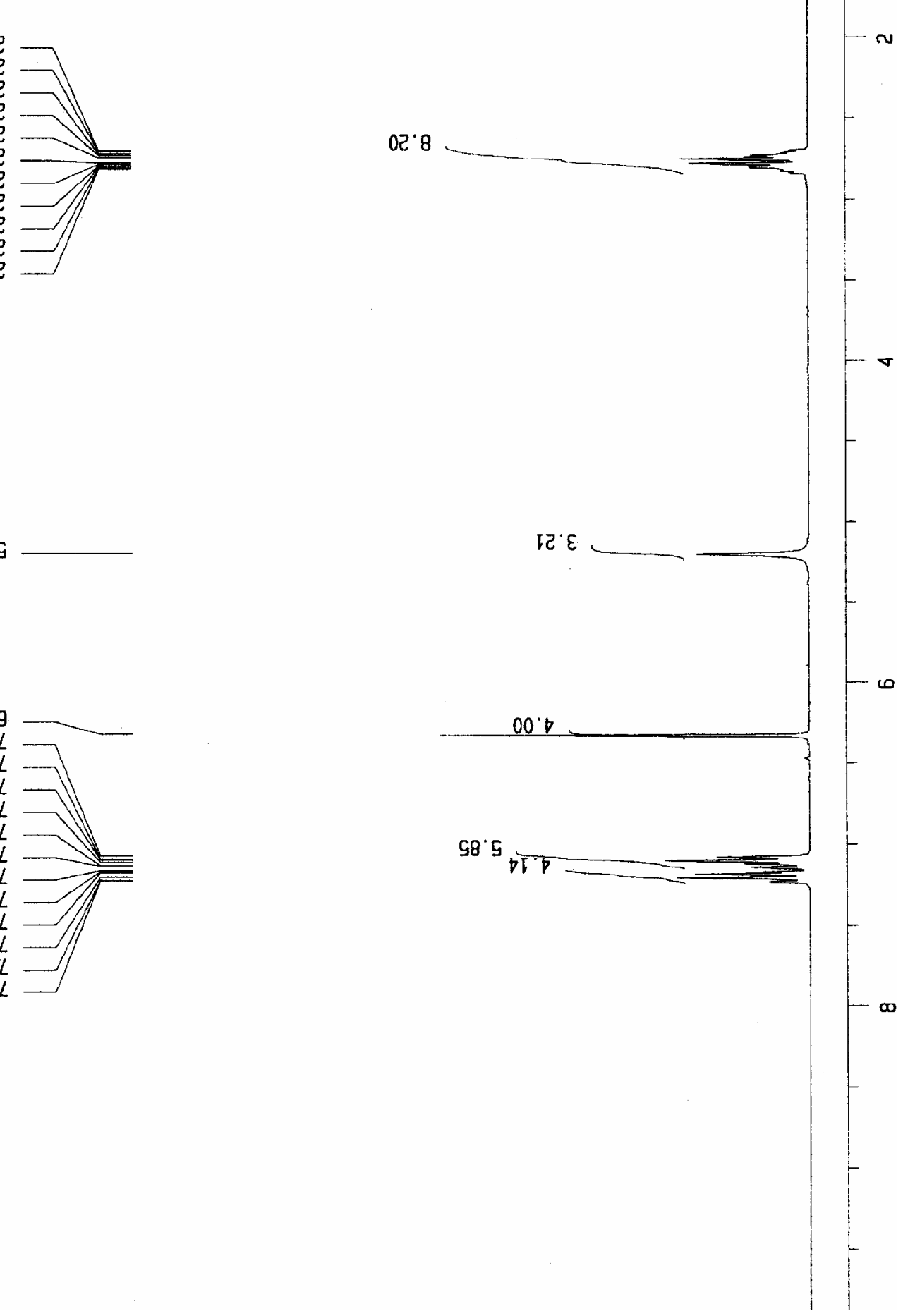


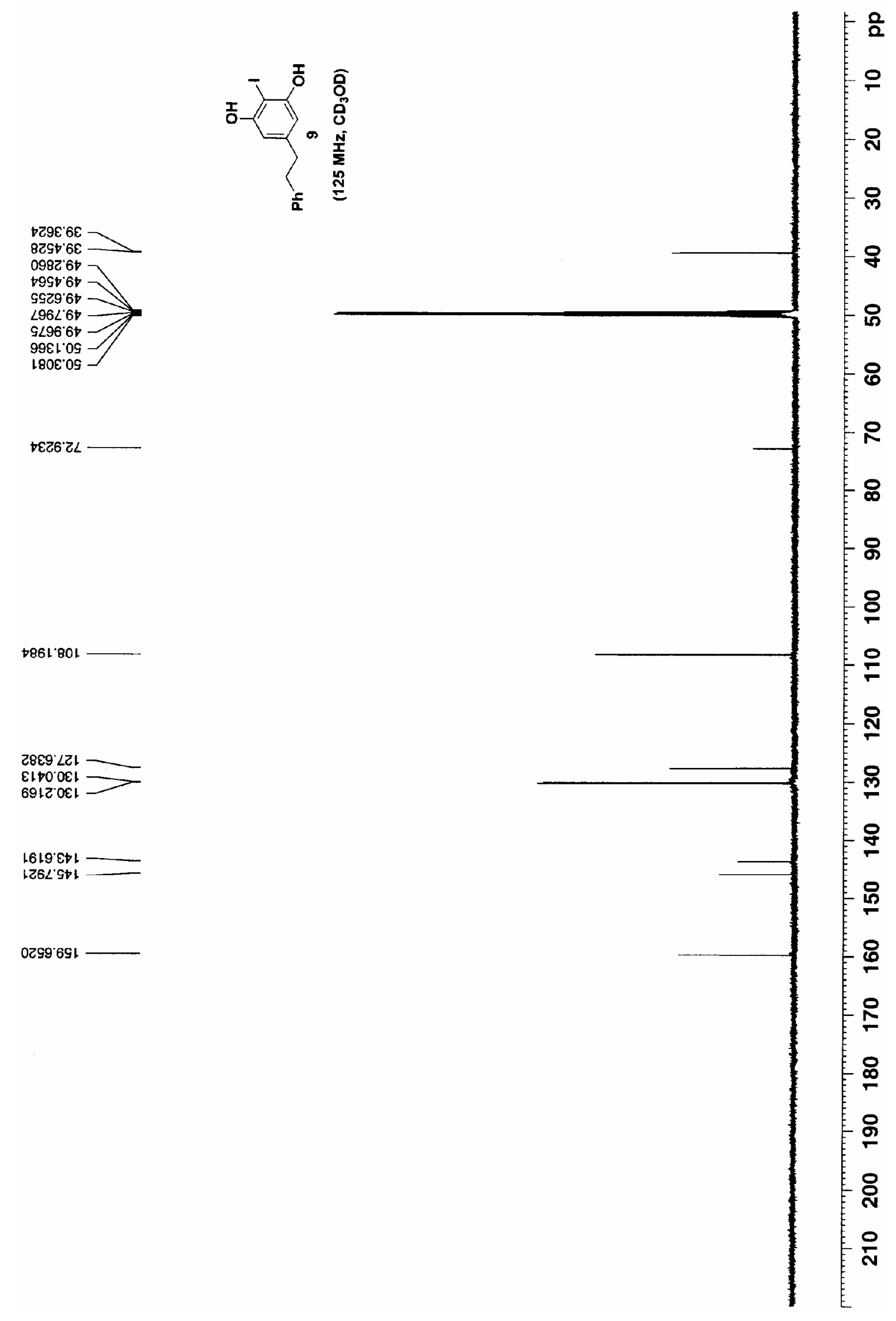




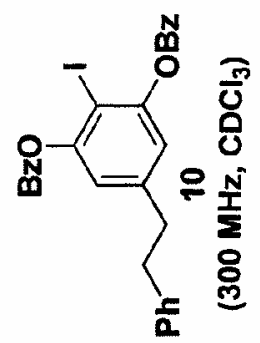

$10 \angle 6^{\circ} \mathrm{C}$

O290 $L$

$\varepsilon 002$ : $L$

ट97ट

E6I2 ' $L$

00b2.

SOLC $L$

IEGZ.

8962.

SBOS.

6ZEG

99ES $L$

869G ' $L$

BIE9 ' $L$

IIE9 $L$

$\angle 6 E 9$ ' $\angle$

LI99' $L$

S989 $L$

$1689^{\circ} L$

I9S2 8

OI92 8

$8 \div 8 \mathrm{C}^{\circ} 8$

จBQ己 8

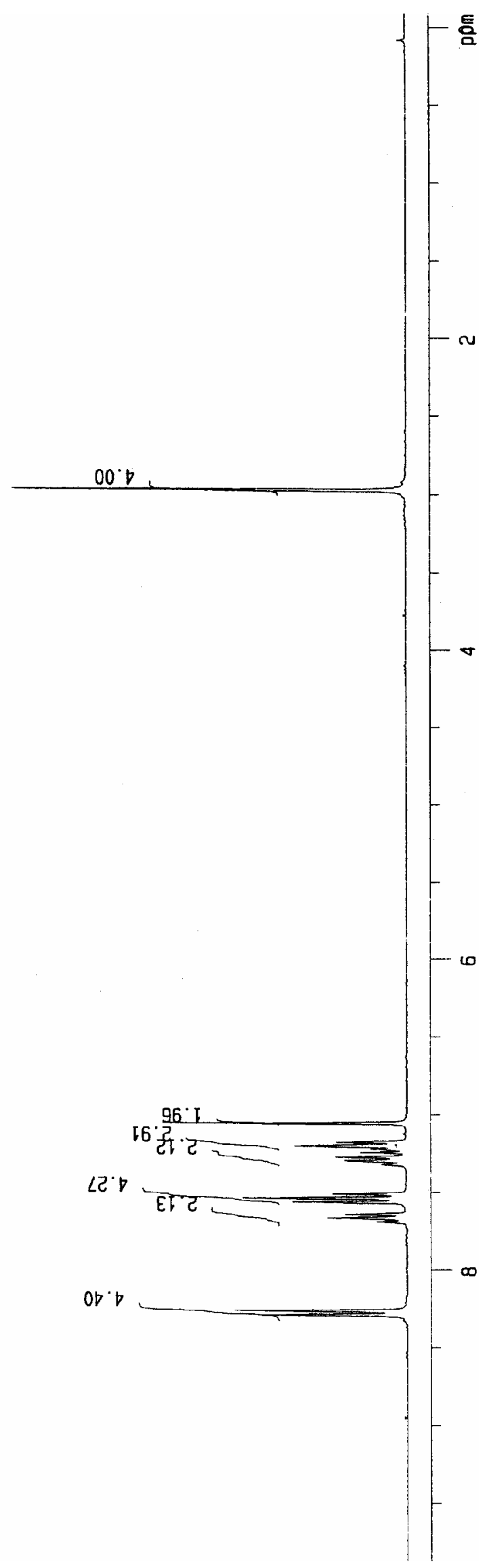



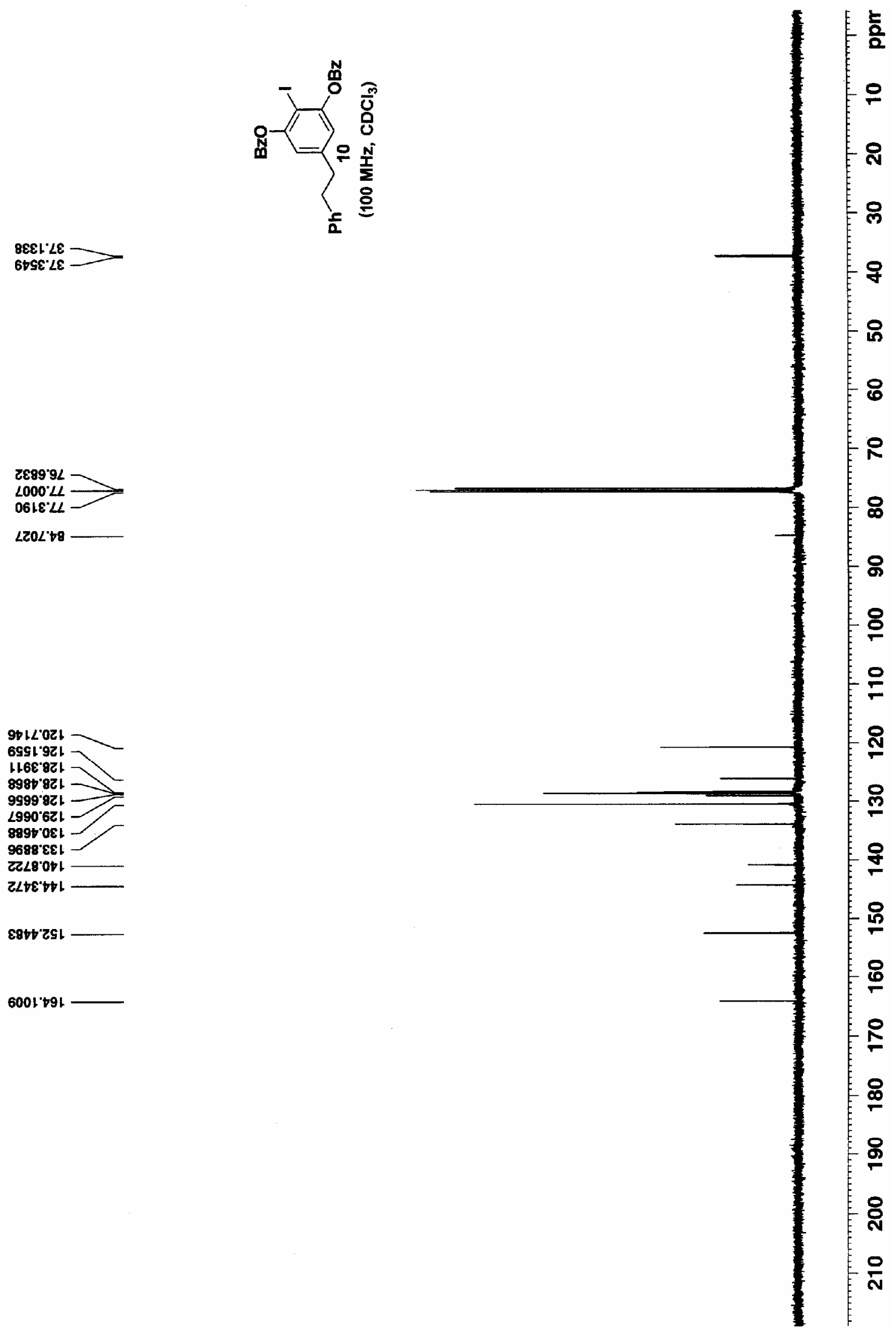

2899'9L

2000 LL

$0618 \angle \longrightarrow$

LZOL't8

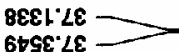

音

กิ

모

䄈

옴

8

?

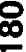

号

:

$\frac{0}{N}$ 
SBE6 0

6656 0

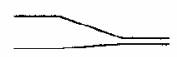

$\varepsilon 6^{\circ} 2$

06EG

$\mid$

$0186^{\circ} \mathrm{C}$

EI
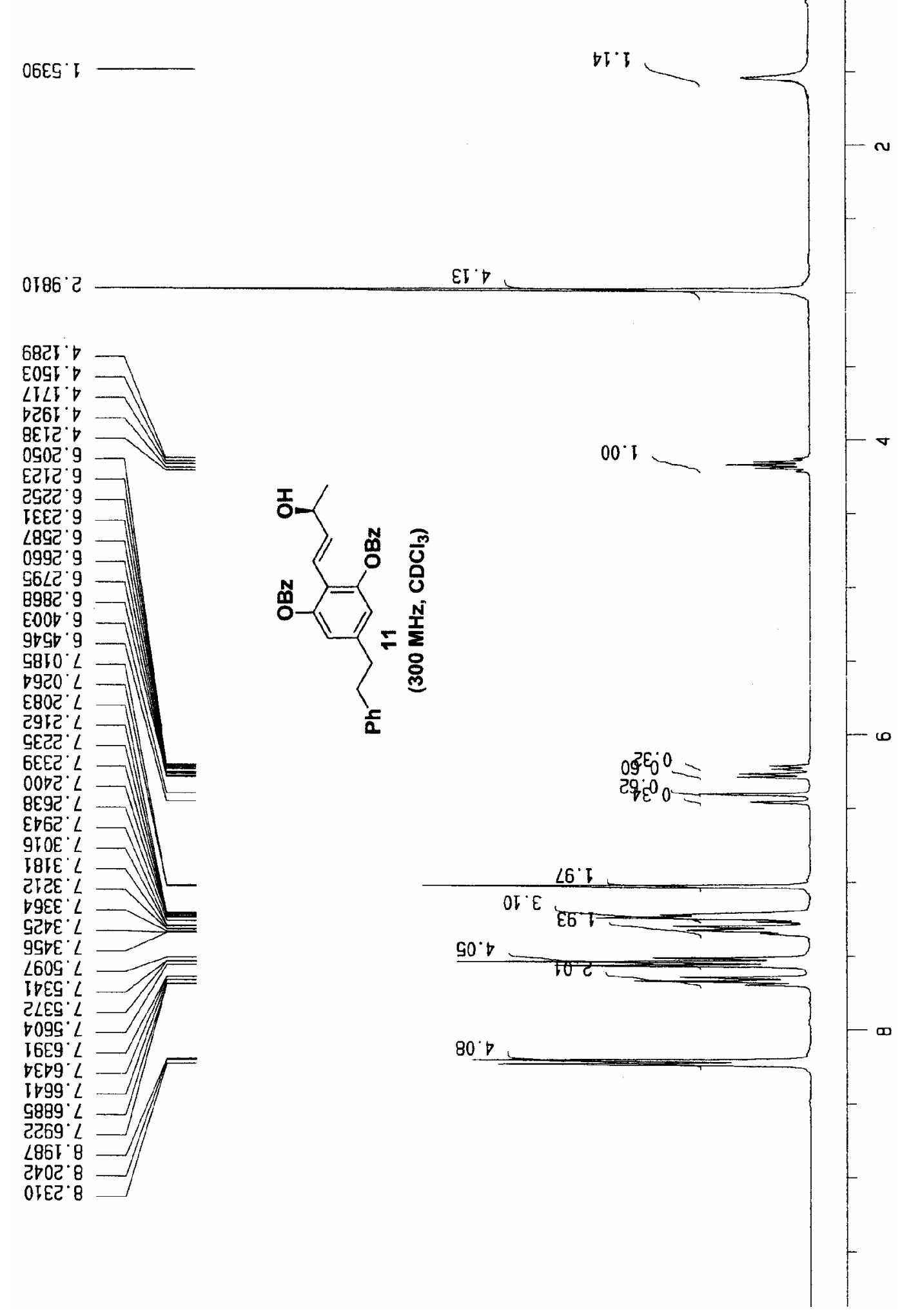

$\sim$ 

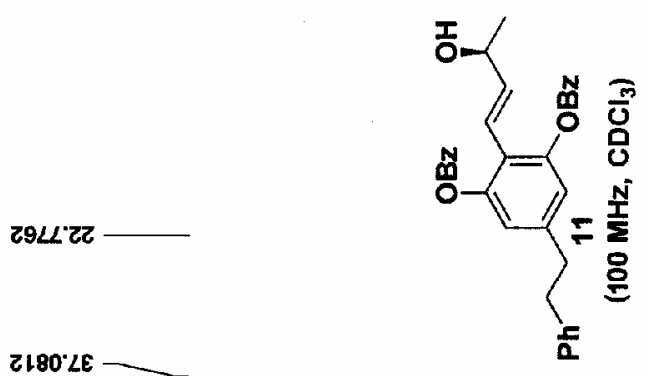

8098 '89

$6189.9 L$

$2000^{\circ} \angle L$

$881 \varepsilon L$

8เ12.8เ

LEZS'0ZL

6859'เ乙L

8810'9et

gL8E'gz।

LO†9'8Z1

9660'6z।

6690.081

Lᄂ४LE\&L

oosz'0t 1

10<0' LD.

$\checkmark \angle 06 C^{\circ} \mathrm{L}$

8E9t'6th

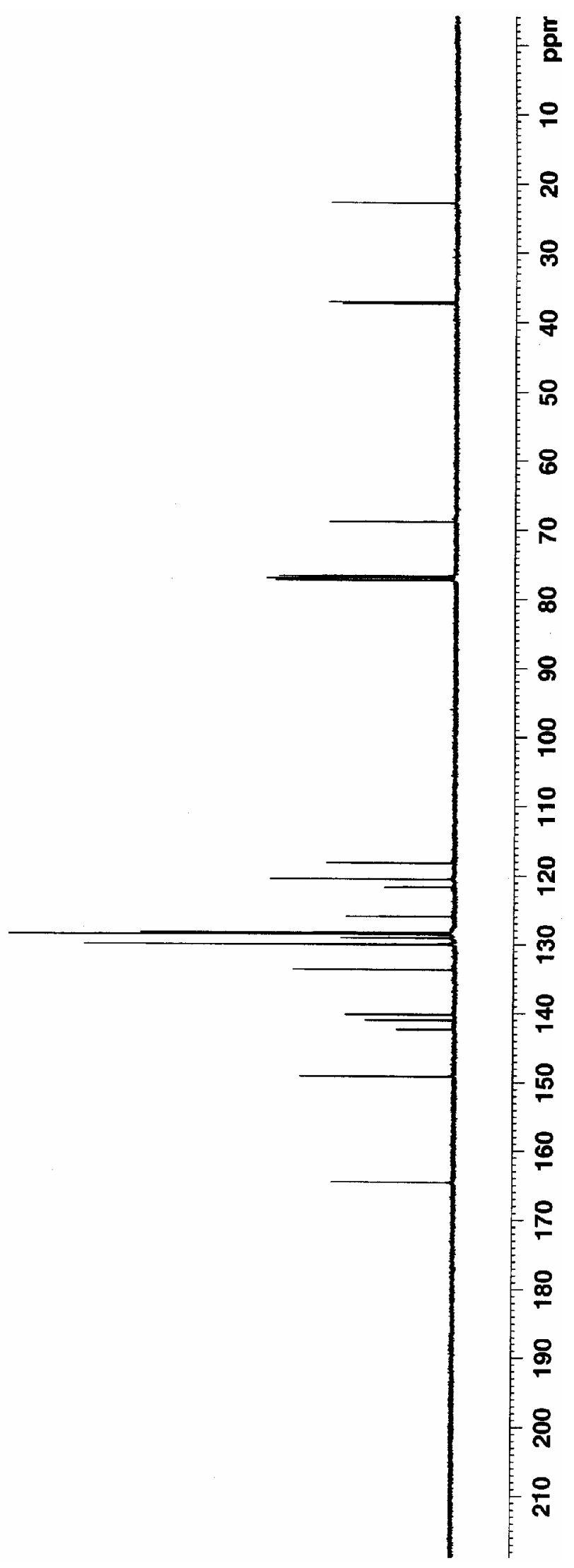



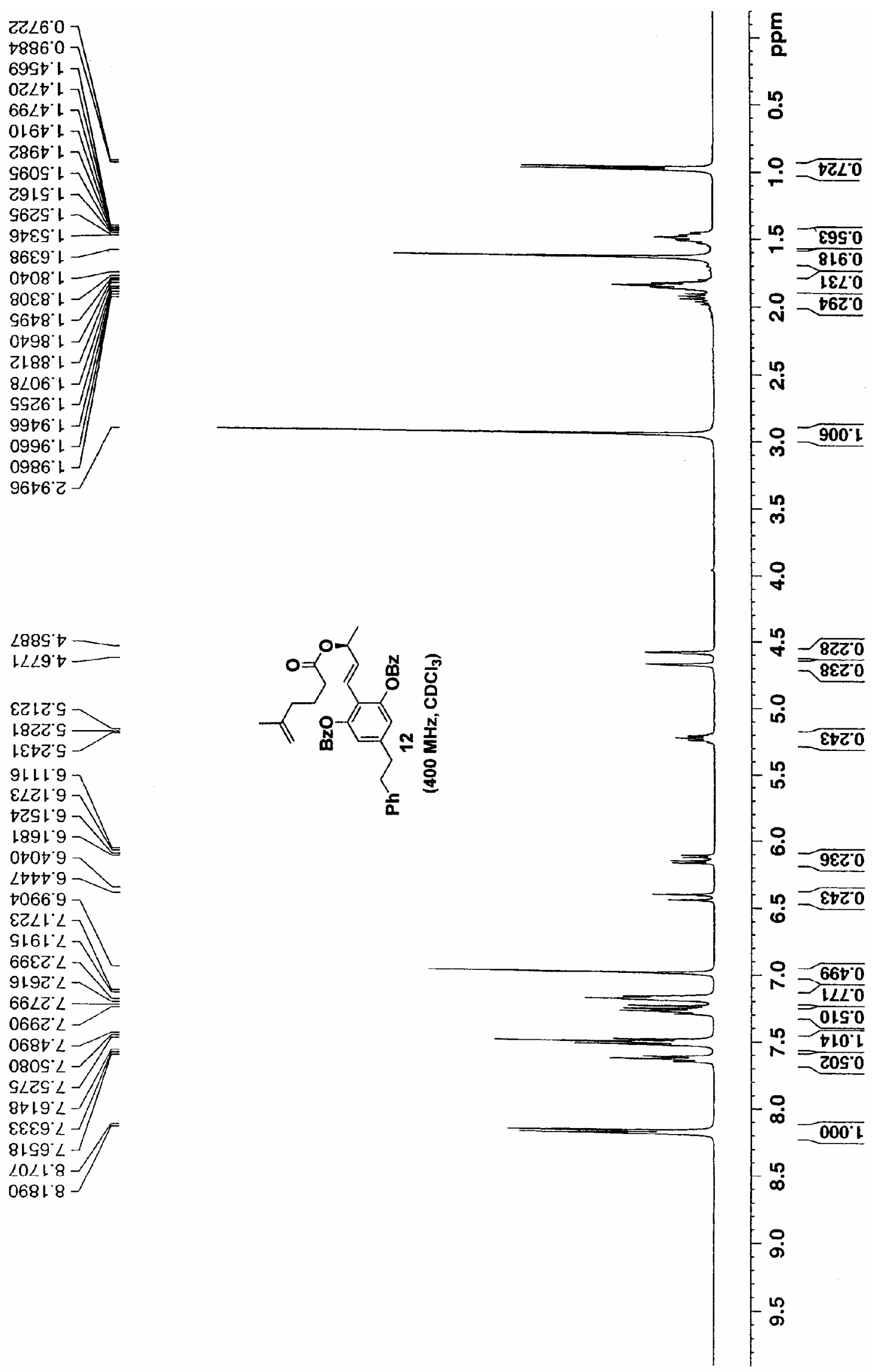

$\angle 889^{\circ} \circ$

EZLZ'S

เ8Z2'S

LEt?'S-

9டL1:97

ELZ1 9

七टSเ'9

$1891^{\circ} 9$

OคOท 9

$\angle \nabla \nabla \nabla 9$

饥6 9

$\varepsilon 2 \angle L^{\circ} L$

SL6! 2

$66 \varepsilon 2 \angle$

$919 \mathrm{C}^{\circ} L$

$66 \angle Z^{\prime} L$

$0662: L$

$068 V^{\circ} L$

0809" $L$

SLCS $\angle$

$8 \nabla 19^{\circ} \mathrm{L}$

8E89 $\mathrm{L}$

$8199 \angle$

101.8

$0681 \cdot 8-$ 

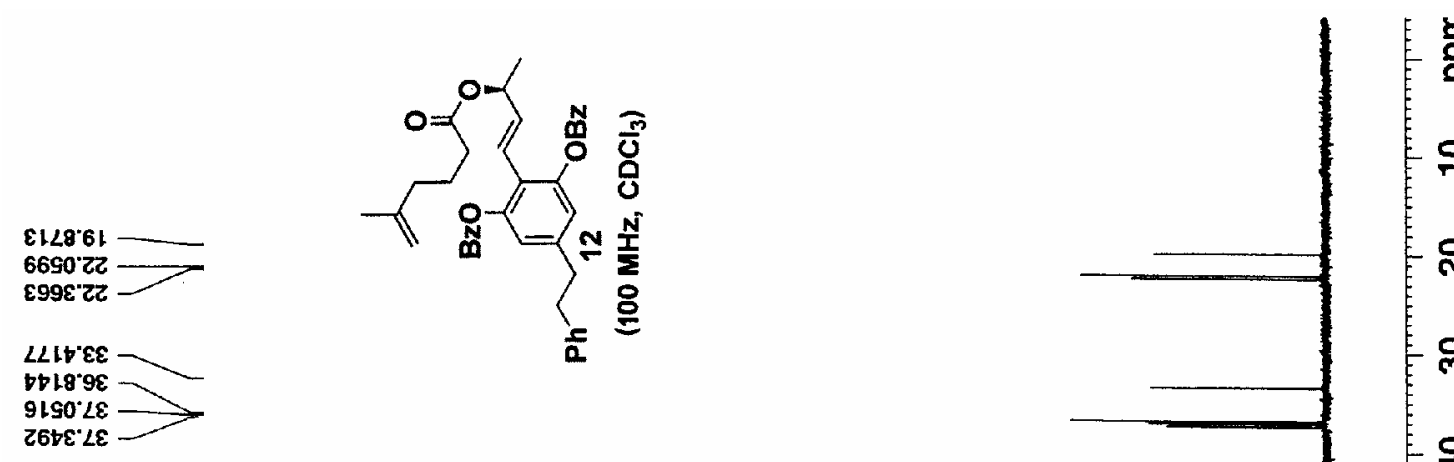

6ट\&Z०

9089 $9 L$

$2666^{\circ} 9 L \longrightarrow$

$6 L 1 \varepsilon " L$

동

능

으

สิ

요

우

요

$\because$

-

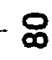

8

8

음

b9LZ0Z

LL9t'OZL

$818 E L Z 1$

0L86.GZL

0862821

टह०ध $8 \mathrm{zL}$

0959'8Z1

$0 \angle 6062$.

$1860 \circ 0 \mathrm{~L}$

6929'8E

68EE SEL

7826001

9E9s'Zb।

oe6t'tol

0z01.6bl.

ย๖8ย"ซgเ

$01<12<1-$

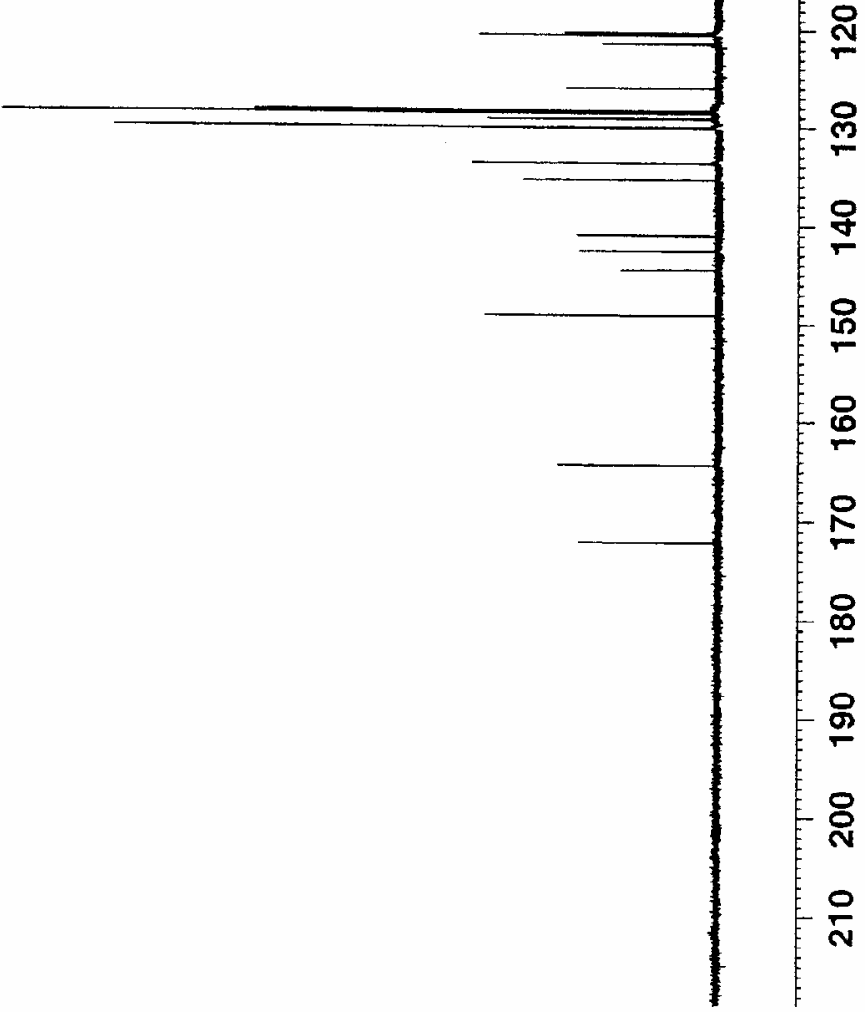



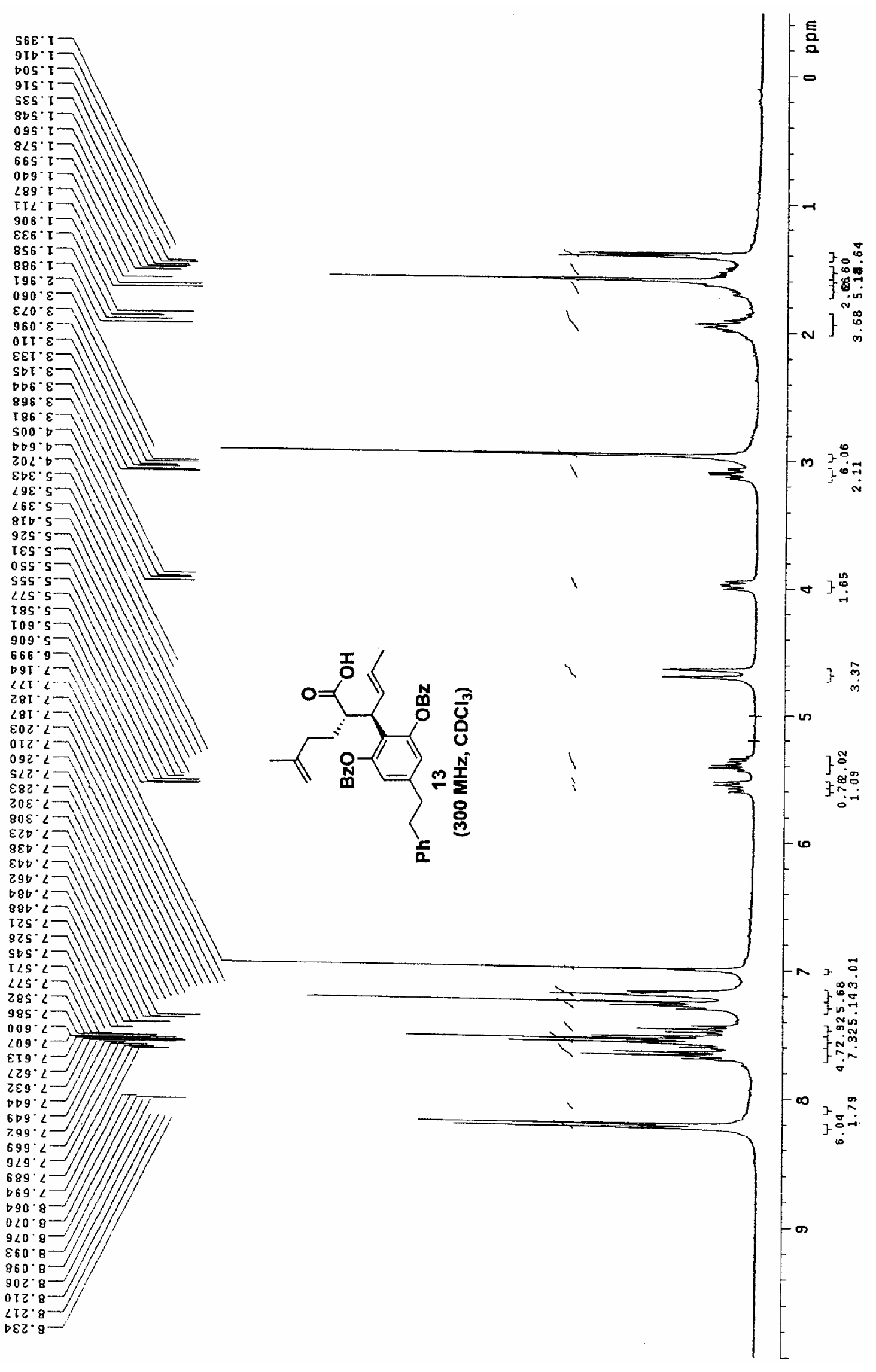


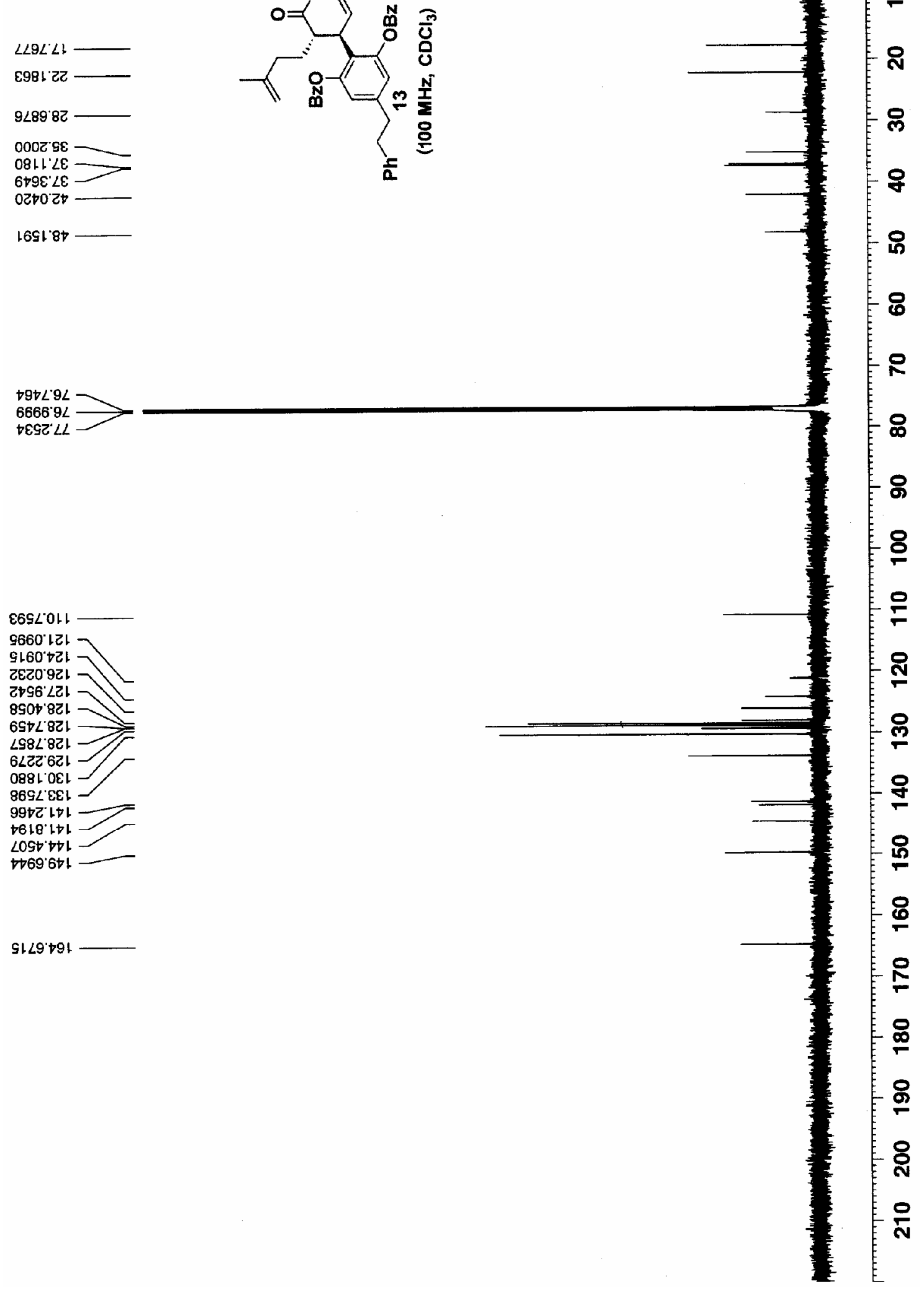




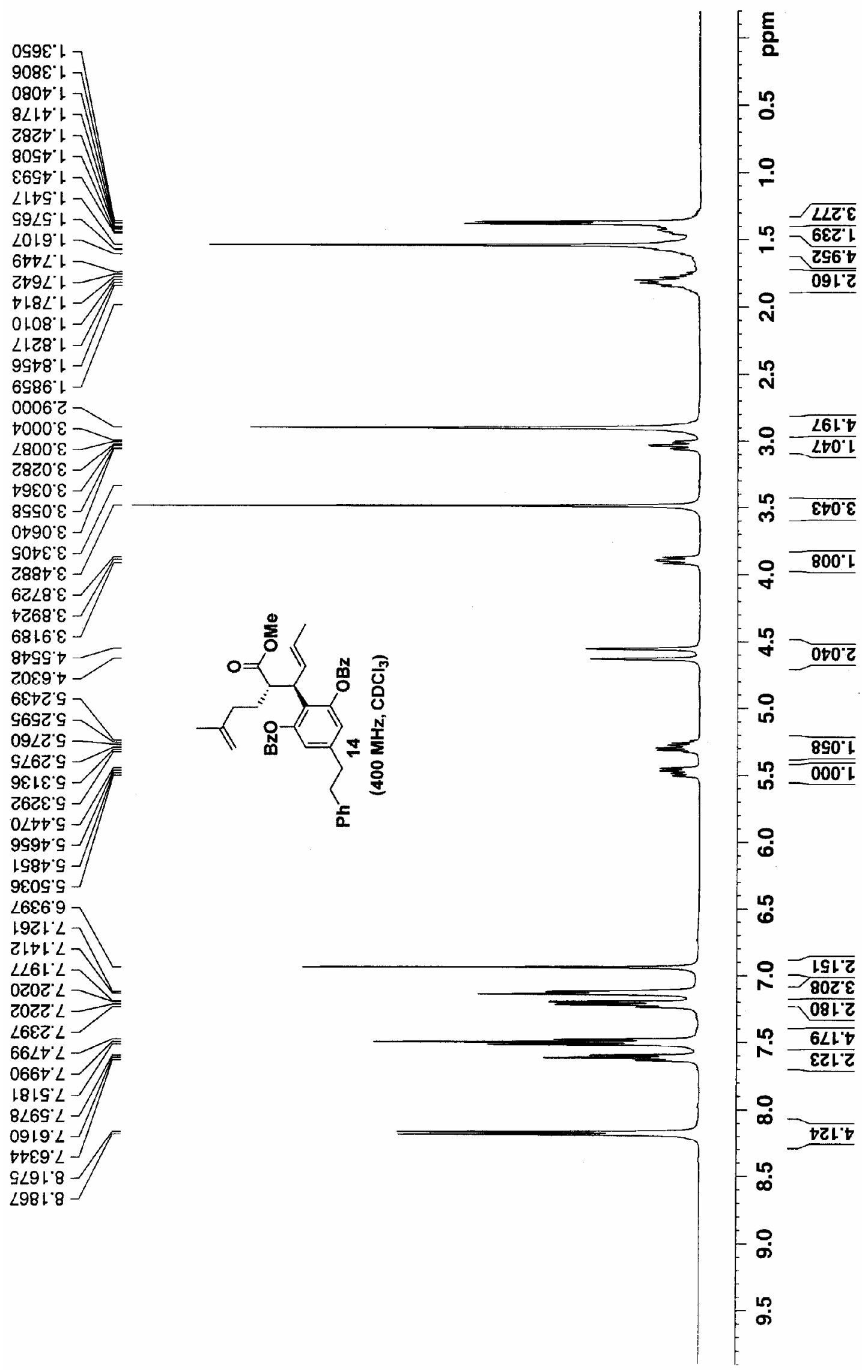




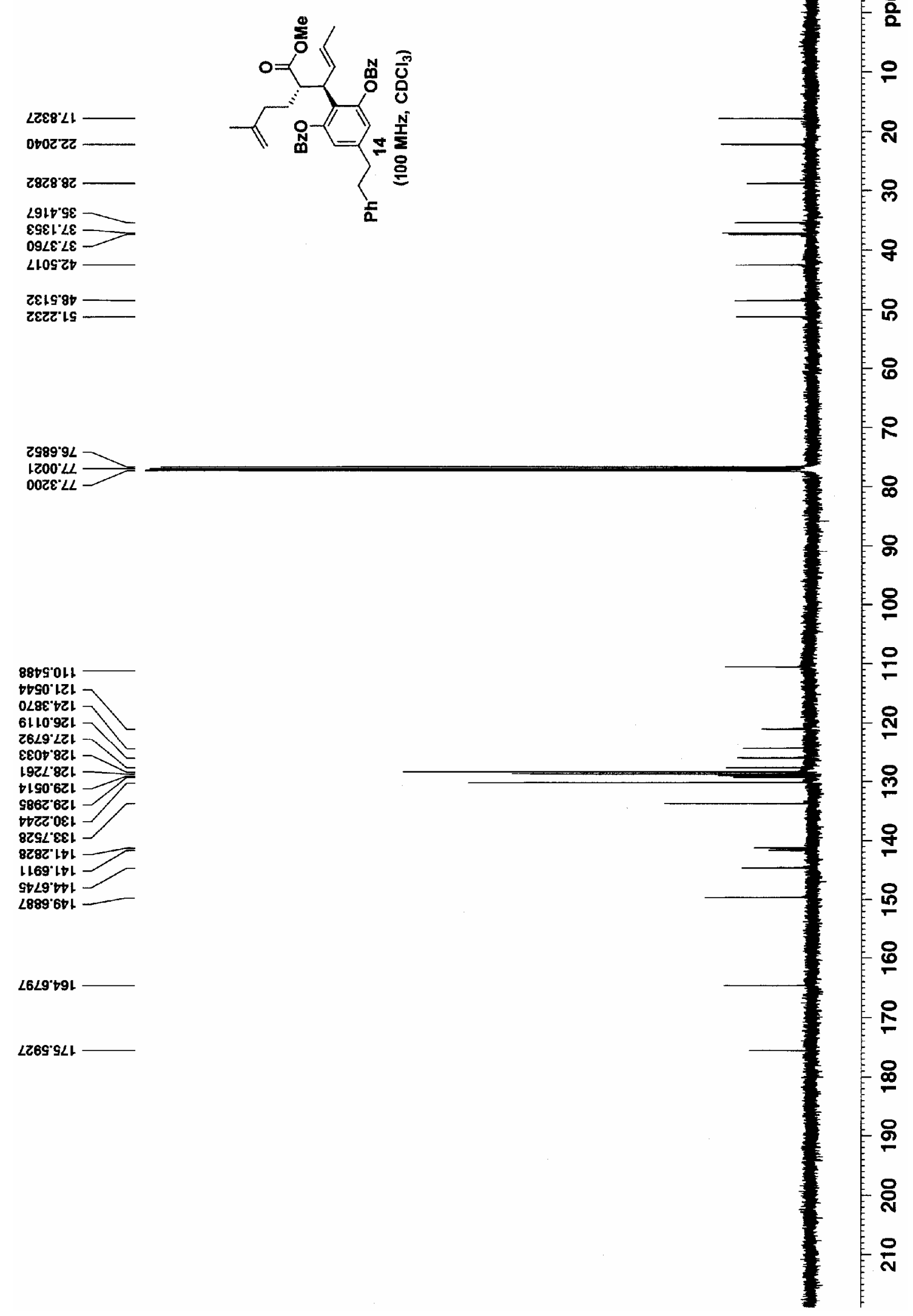




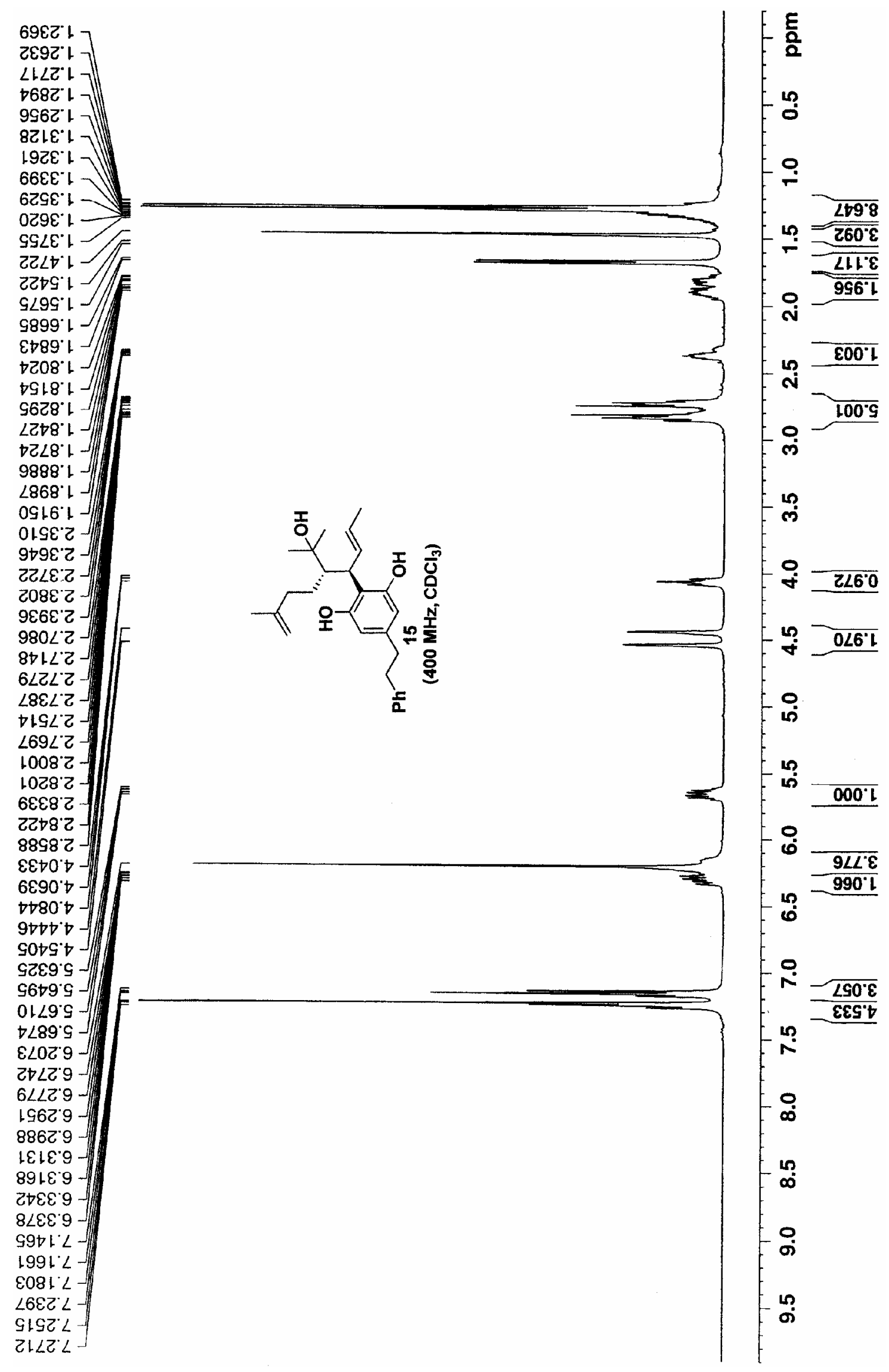



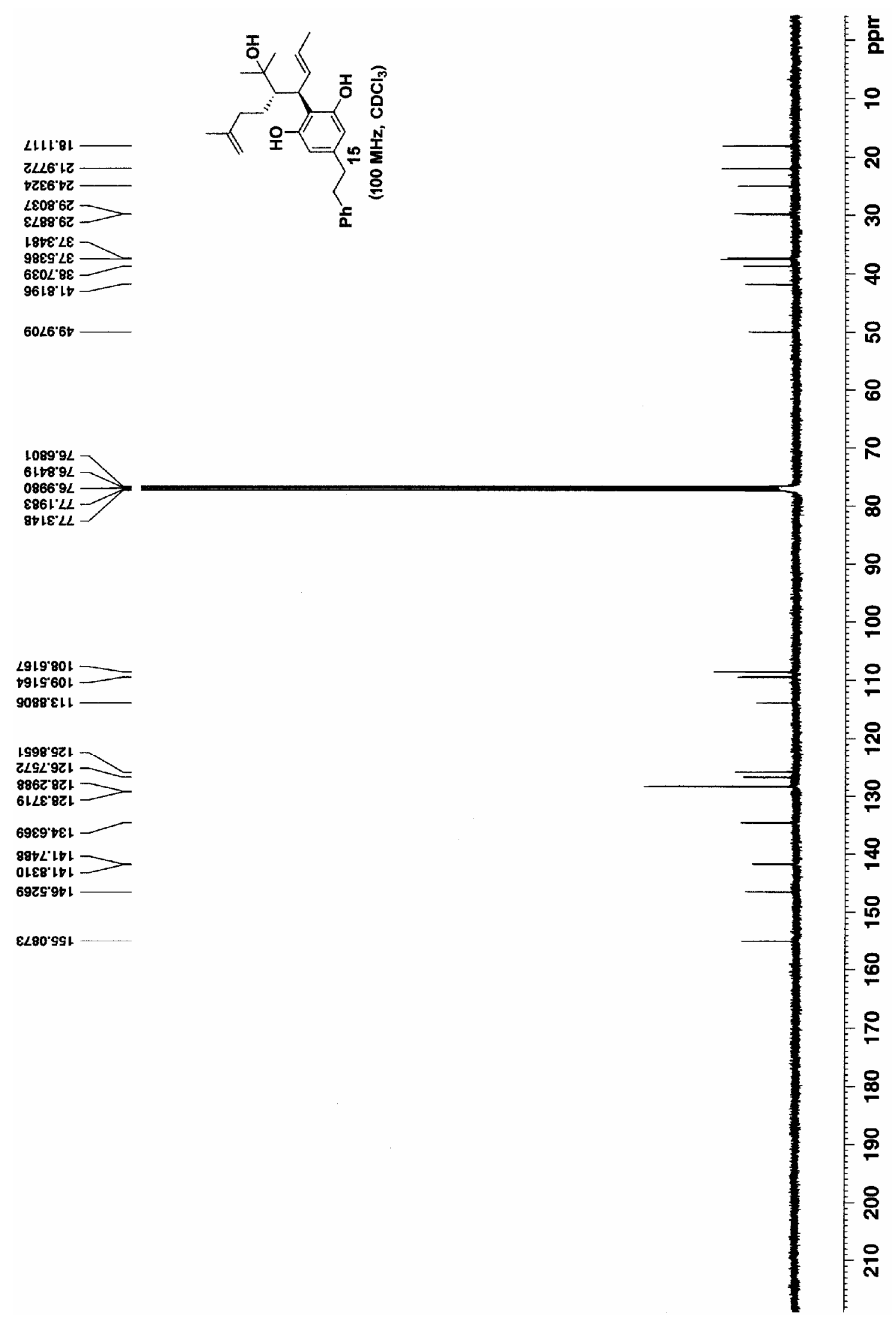

$61+8.92$

0866.92

E86I'LL

8tIE:LL -

$\angle 919^{\circ} 801-$
$+919^{\circ} 601-$

9088'EL।

IS98.GZI

ZL9L9ZL

8862 821 $\longrightarrow$

6LLE'8ZL

$6989^{\circ} \circ \varepsilon 1$

$88 \square L\llcorner$ L

0LE8เt। $>$

69zs'9bl

EL80'SSL 


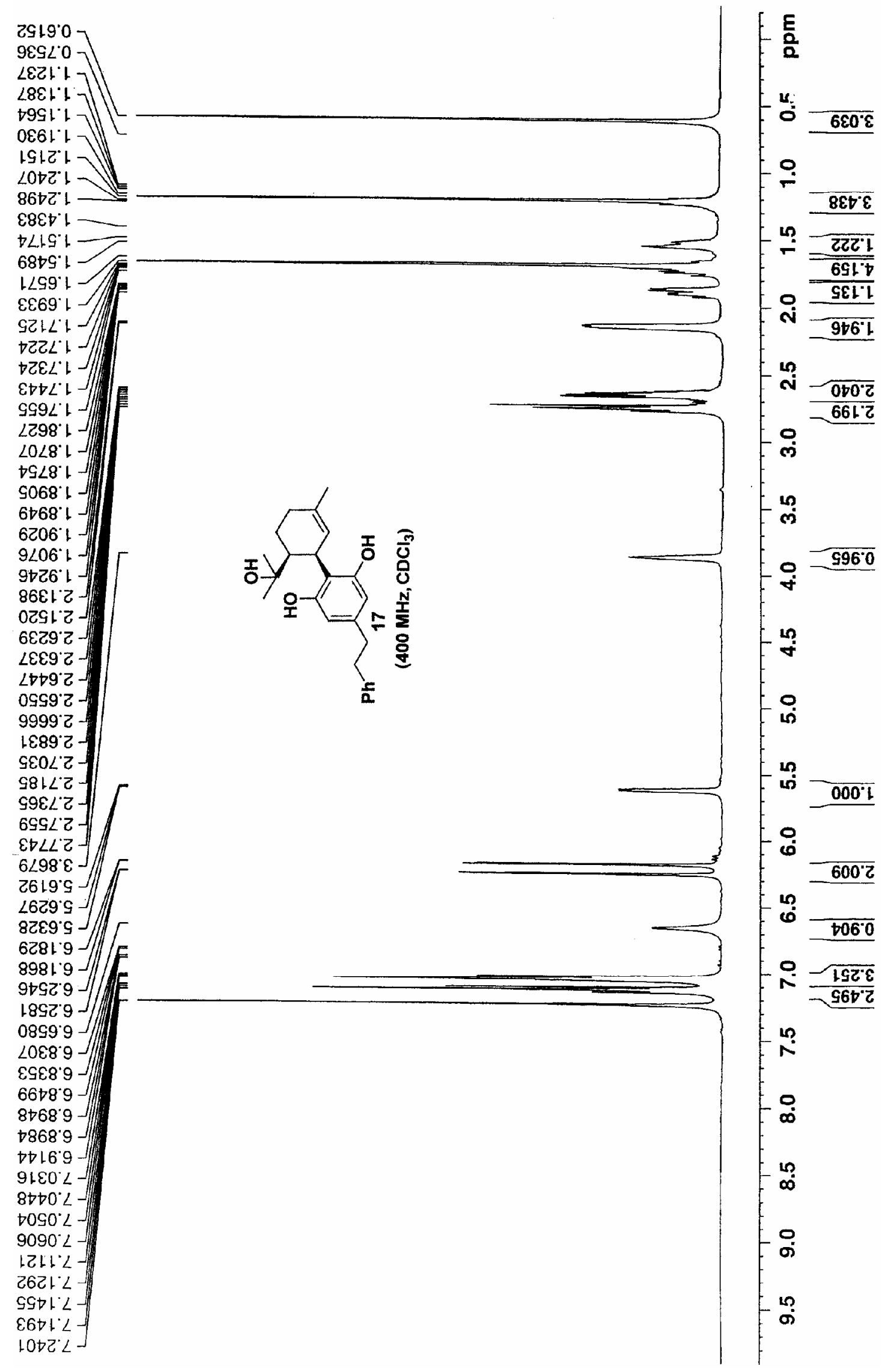


\&829' 22

9ZL6'ZZ

$8192 . \textrm{ }$

99L8'เE

E LIO'ZE

$\checkmark 990^{\circ} \varepsilon \varepsilon$

乙6เE: $\angle \mathcal{E}=$

$8<1909$

$6+t \angle \cdot 9$

$2666^{\circ} 9 \mathrm{~L}$

LZSZ LL

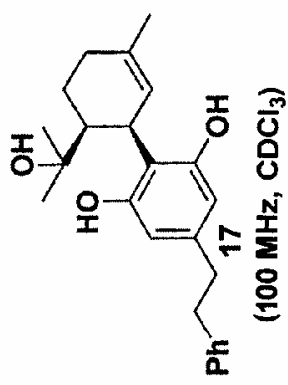

S199.01

E68Lㅇㄴ

098Z"Zト.

2609 92

$\angle 889^{\circ} \mathrm{GZ}$.

$1.08 \mathrm{SZ}$

10EZ'BटL

IHLBZL

$\angle 8+1 \cdot 6 E \mid$

$60+8 \cdot 1+1-$

$6+\angle 6 \cdot 2+1=$

$9826^{\circ}+91$

LITE' LSL

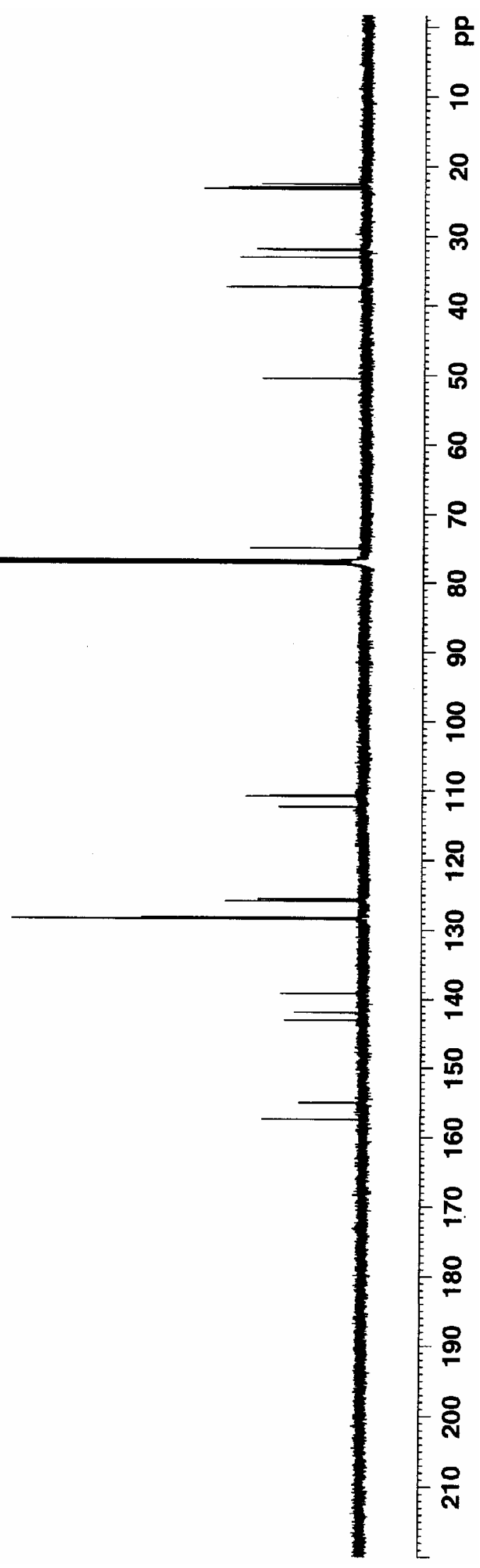

๑

8

응 

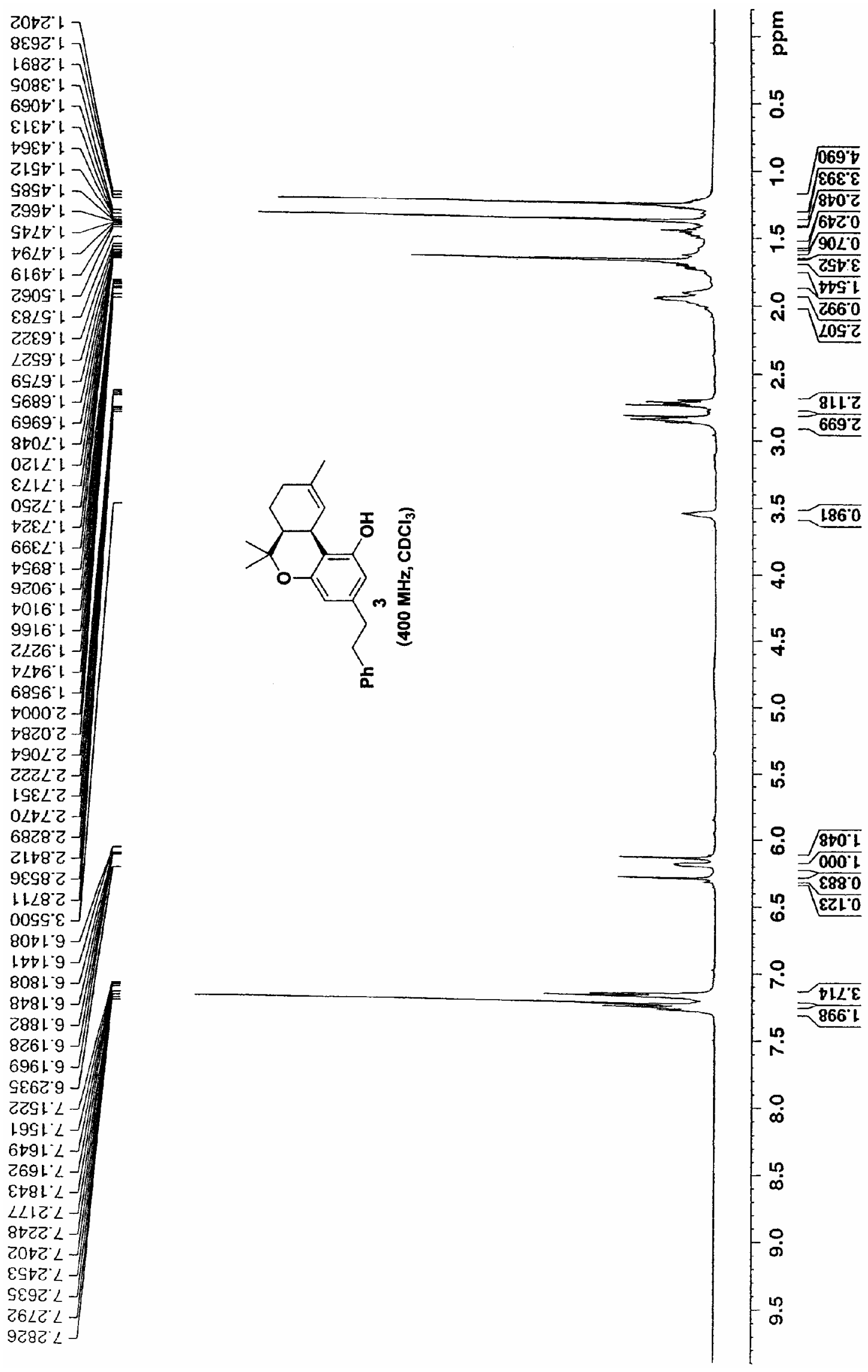
$29 \angle 0.12$

$8 z 80$ to

Z8ZE' $9 Z$

008L. 0

$\angle 916.1 \varepsilon$

¿ $6 \angle 2 \angle \varepsilon$

ISZB' $\angle E$

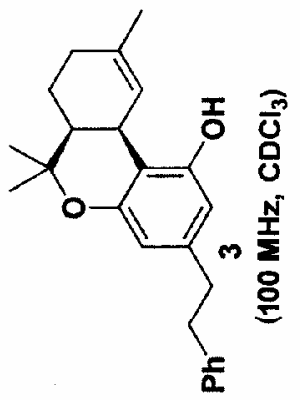

6 เto 0 -

(2)

을

으

สิ

요

우

in

8

$R$

ळ

8

응

0เZع $80 \mathrm{~L}-$

6เ SZOเ

618E.01 =

89๕ट टZ।

ZL8Z'9Zト

$6 \angle 88^{\circ} 8 \mathrm{ZL}$

ES9S'SEL

8992' $|t|$

EsLtZtセt

$8 \varepsilon+\hbar \cdot+5 !$

8ELESGL

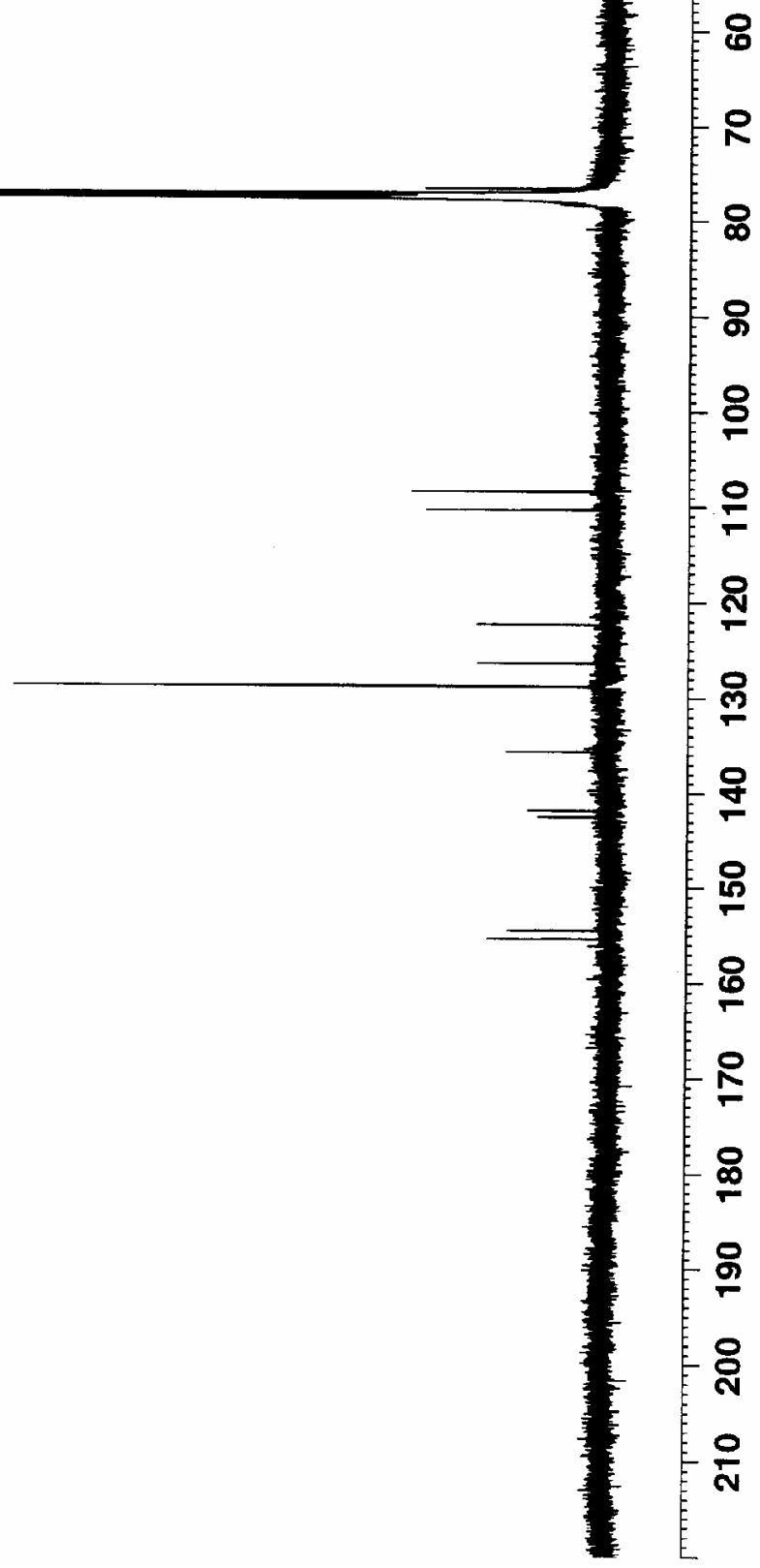

